\title{
Assays to Interrogate the Ability of Compounds to Inhibit the AF-2 or AF-1 Transactivation Domains of the Androgen Receptor
}

\author{
Ashley T. Fancher, ${ }^{1}$ Yun Hua, ${ }^{1}$ \\ Christopher J. Strock, and Paul A. Johnston ${ }^{1,3}$ \\ ${ }^{1}$ Department of Pharmaceutical Sciences, School of Pharmacy, \\ University of Pittsburgh, Pittsburgh, Pennsylvania. \\ ${ }^{2}$ Cyprotex US, Watertown, Massachusetts. \\ ${ }^{3}$ Head and Neck Cancer, and Skin Cancer Specialized Programs \\ of Research Excellence, University of Pittsburgh Hillman Cancer \\ Center, Pittsburgh, Pennsylvania.
}

\section{ABSTRACT}

Prostate cancer is the leading cause of cancer and second leading cause of cancer-related death in men in the United States. Twenty percent of patients receiving the standard of care androgen deprivation therapy (ADT) eventually progress to metastatic and incurable castration-resistant prostate cancer (CRPC). Current FDA-approved drugs for CRPC target androgen receptor (AR) binding or androgen production, but only provide a 2- to 5-month survival benefit due to the emergence of resistance. Overexpression of AR coactivators and the emergence of $A R$ splice variants, both promote continued transcriptional activation under androgen-depleted conditions and represent drug resistance mechanisms that contribute to CRPC progression. The $A R$ contains two transactivation domains, activation function 2 (AF-2) and activation function 1 (AF-1), which serve as binding surfaces for coactivators involved in the transcriptional activation of $A R$ target genes. Full-length AR contains both AF-2 and $A F-1$ surfaces, whereas $A R$ splice variants only have an $A F-1$ surface. We have recently prosecuted a high-content screening campaign to identify hit compounds that can inhibit or disrupt the protein-protein interactions (PPIs) between AR and transcriptional intermediary factor 2 (TIF2), one of the coactivators implicated in CRPC disease progression. Since an ideal inhibitor/ disruptor of AR-coactivator PPIs would target both the AF-2 and $A F-1$ surfaces, we describe here the development and validation of five AF-2- and three AF-1-focused assays to interrogate and prioritize hits that disrupt both transactivation surfaces. The assays were validated using a test set of seven known AR modulator compounds, including three $A R$ antagonists and one androgen synthesis inhibitor that are FDA-approved ADTS, two investigational molecules that target the $N$-terminal domain of $A R$, and an inhibitor of the Hsp90 (heat shock protein) molecular chaperone.

Keywords: androgen receptor activation function domains, castration-resistant prostate cancer, mammalian 2-hybrid assays

\section{INTRODUCTION}

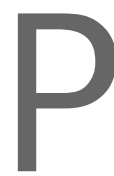

rostate cancer $(\mathrm{PCa})$ is the second leading type of cancer in men in the United States. ${ }^{1}$ Twenty percent of PCa patients develop a terminal form of the disease termed castration-resistant prostate cancer (CRPC) that results in nearly 30,000 deaths per annum in the United States. ${ }^{1,2}$ The mean survival for patients diagnosed with metastatic CRPC is only 9-13 months. ${ }^{2}$ The standard of care treatment options for patients with PCa are surgery and radiation for more localized tumors, and androgen deprivation therapy (ADT) for recurrent or metastatic tumors. ${ }^{3,4}$ Since CRPC cells continue to rely on androgen receptor (AR) signaling for growth and proliferation, ADT drugs modulate AR functions either directly by antagonizing androgen binding to the receptor or indirectly by blocking androgen synthesis. ${ }^{5}$ Unfortunately, ADT drugs also produce adverse events such as osteoporosis, loss of muscle, and muscle weakness. Furthermore, disease flare-up associated with ADT can produce bone pain in patients whose tumors have metastasized to bone tissue, and bursts of tumor growth can also lead to spinal cord compression, or even death. ${ }^{6}$ Around $80 \%$ of patients receiving ADT progress to CRPC. ${ }^{7}$ The AR antagonist enzalutamide and the androgen synthesis inhibitor abiraterone, the two drugs most recently approved by the FDA for CRPC, provide only a modest 3- to 5-month patient survival benefit, ${ }^{8,9}$ underscoring the urgent need to identify and develop novel drugs for CRPC treatment. Multiple mechanisms of resistance arise in CRPC cells to render ADT drugs ineffective. ${ }^{10-12}$ AR-dependent resistance mechanisms include AR amplification, intratumoral androgen synthesis, AR mutations that lead to receptor promiscuity, and the emergence of AR splice variants 
such as androgen receptor splice variant 7 (AR-V7), which have lost the ligand-binding domain (AR-LBD) in the Cterminus of the receptor and therefore do not respond to the current antiandrogens. ${ }^{10-12}$ Furthermore, overexpression of AR coactivators also contributes to poor CRPC patient outcomes by increasing AR transcriptional activity under androgen-depleted conditions. ${ }^{13}$ Coactivator interactions with both the full-length AR (ARFL) and splice variants like AR-V7 compensate for low cellular dihydrotestosterone (DHT) levels after ADT treatment and result in persistent transcriptional activity. ${ }^{13-16}$

Under normal androgen-dependent conditions, AR transcriptional activation begins after ligand-activated AR traffics from the cytoplasm into the nucleus, binds to DNA at specific androgen response elements (AREs) on the promoters of AR target genes, and recruits coactivators that enable histone remodeling, additional coactivator recruitment, and subsequent assembly of the transcriptional machinery. ${ }^{17}$ AR target genes associated with cellular biosynthesis, survival, and proliferation contribute to PCa disease progression into CRPC. ${ }^{18}$ Small molecule drugs that could inhibit AR transcriptional activity represent an alternate mechanism to existing ADTs for the treatment of CRPC. One strategy to inhibit AR-mediated transcriptional activity would be to find small molecules that prevent or disrupt the protein-protein interactions (PPIs) between AR and its coactivators, and thereby block transcription at target gene AREs. ${ }^{13,16,19-21}$ Elevated expression of several AR coactivators have been observed in relapsed PCa patients, including transcriptional intermediary factor 2 (TIF2), steroid receptor coactivator (SRC)-1, RAC3, p300, CBP, Tip60, and ARA 70. ${ }^{13,16,19-21}$ TIF2 (TIF2/SRC-2/ NCoA-2/GRIP1) represents a promising drug target for CRPC because TIF2 overexpression occurs in more advanced stages of PCa and increased TIF2 levels correlate with biochemical recurrence in $\mathrm{PCa}$ patients. ${ }^{13}$ Small interfering RNA (siRNA) knockdown of TIF2 reduced AR target gene expression and slowed the proliferation of androgen-dependent and androgen-independent PCa cells. ${ }^{19}$ The cumulative evidence implicating TIF2 in the progression of PCa into CRPC prompted the development and implementation of a highcontent screening (HCS) PPI biosensor (PPIB) assay to identify small molecules that could inhibit the formation of agonistinduced AR-TIF2 PPIs or that could disrupt pre-existing ARTIF2 complexes. ${ }^{22-25}$

The AR-TIF2 PPIB assay used two adenovirus constructs to coexpress AR-LBD residues 662-919 as a chimera with red fluorescent protein (AR-RFP), and TIF2 residues $725-840$ as a chimera with green fluorescent protein (TIF2-GFP). ${ }^{22-25}$ In cells infected with only the AR-RFP biosensor, which also contains nuclear import and export sequences, AR-RFP expression is localized predominantly to the cytoplasm, but shifts to the nucleus after exposure to DHT (Supplementary Fig. S1). The TIF2 adenovirus contains the receptor interacting domain box III LXXLL (coactivator leucine rich binding motifs) motif that mediates binding to ligandactivated AR-LBD and high-affinity nuclear localization sequence (NLS), and nucleolar localization sequence (NoLS) derived from HIV Rev, which tethers the TIF2-GFP biosensor in the nucleolus of the cell. ${ }^{22-25}$ In cells infected with the TIF2GFP biosensor alone, TIF2-GFP expression is restricted to the nucleolus within the nucleus irrespective of DHT exposure (Supplementary Fig. S1). In untreated cells coinfected with both AR-RFP and TIF2-GFP biosensors, TIF2-GFP expression is localized only in the nucleoli and AR-RFP expression is predominantly cytoplasmic, but upon exposure to DHT, the AR-RFP biosensor traffics into the nucleus where the PPIs between the AR and TIF2 components result in their colocalization within the nucleoli, as indicated by the bright yellow nucleolar puncta of the FITC and Texas Red composite images (Supplementary Fig. S1). ${ }^{22-25}$ Three libraries totaling 143,000 compounds were screened in an AR-TIF2 PPIB HCS campaign to identify concentration-dependent inhibitors and/or disruptors of AR-TIF2 PPIs. ${ }^{25}$ The AR-TIF2 PPI hits were then triaged in counter screens designed to identify and eliminate compounds that interfered with the PPIB assay format, nonspecifically blocked nuclear receptor (NR) trafficking to the nucleus, or reduced AR expression and/or restricted its localization to the cytoplasm. ${ }^{26-32}$

The AR has two major transactivation domains, activation function $2(\mathrm{AF}-2)$ and activation function 1 (AF-1), which serve as binding surfaces for coactivators that activate AR gene transcription. ${ }^{14,15,33-36}$ The AF-2 binding surface is formed by helix 12 in the C-terminal domain of the receptor after DHT binds to the AR-LBD, ${ }^{35-39}$ and enables the interactions between $A R$ and the cohorts of coactivators that modulate AR transcriptional activity. The AF-2 surface interacts with coactivators containing binding motifs encoded by LXXLL and/or FXXLF sequences. ${ }^{14,37-39}$ The AF-1 binding surface is located in the intrinsically disordered N-terminal domain of AR (AR-NTD) where the transactivation unit 1 (TAU1) and transactivation unit 5 (TAU5) subregions are as important for ligand-independent transactivation. ${ }^{14,34,40,41}$ TAU5 is required for the recruitment of p160 SRCs 1-3, of which TIF2 (SRC2) is a member. ${ }^{14,34,40,41}$ The AR-NTD AF-1 region is transcriptionally active in the absence of androgens, and AR-NTD constructs lacking an AR-LBD activate reporter genes to the same degree as AR-FL in the presence of ligand. ${ }^{14,15,34,42,43}$ The AF-1 surface is particularly important 
to the transcriptional activity of AR splice variants, which lack a LBD and AF-2 surface. ${ }^{44-46}$ AF-1 containing AR-V7 splice variants, which are upregulated in CRPC patients who have relapsed on $\mathrm{ADT}$, also require coactivators to activate gene transcription. ${ }^{14,15,34,40,42}$

Since the design of the AR-TIF2 PPIB assay might be biased toward finding hits that preferentially inhibited or disrupted DHT-induced AR-LBD AF-2 surface binding with TIF2 Box III LXXLL motifs, ${ }^{22-25}$ we developed a panel of AF-2- and AF-1focused assays to characterize the transactivation domain profiles of our hits and to prioritize those that might be effective against both AR-FL and AR splice variants. AF-2focused assays include mammalian 2-hybrid (M2H) PPI assays between the AR-LBD and the $\mathrm{p} 160$ coactivators TIF2 and SRC-1, a PSA6.1-Luc (luciferase) reporter assay conducted in a PC3 cell line stably expressing AR-FL-GFP, an AR-LBD tritiated dihydrotestosterone $\left(\mathrm{H}^{3}-\mathrm{DHT}\right)$ radio ligand binding assay, and an AR-LBD TIF2 Box III-LXXLL peptide binding assay. The AR-NTD AF-1 surface-focused assays include a constitutive pGAL4 (DNA-binding domain of the Gal4 protein derived from yeast)-AR-NTD transactivation assay and two reporter assays developed in PC3 cells that stably express AR-V7-GFP, a PSA6.1-Luc, and a ubiquitin-conjugating enzyme E2C (UBE2C)-Luc reporter assay. This article describes the development and optimization of the AF-2 and AF-1 assays and their validation using seven known AR modulator compounds, including four FDA-approved ADTs, two investigational molecules that target the AR-NTD, and an inhibitor of the Hsp90 (heat shock protein) molecular chaperone (Supplementary Fig. S2).

\section{MATERIALS AND METHODS}

\section{Reagents}

Formaldehyde, DHT, flutamide, bicalutamide, enzalutamide (MDV3100), EPI-001, abiraterone acetate, 17-AAG (17-allylaminogeldanamycin), and Tris(2-carboxyethyl) phosphine hydrochloride (TCEP) were purchased from Sigma-Aldrich (St. Louis, M0). Compound 10, 2-[(isoxazol-4-ylmethyl)thio]1-(4-phenylpiperazin-1-yl)ethanone ${ }^{47-49}$ was kindly provided by Dr. Zhou Wang in the Department of Urology of the University of Pittsburgh Hillman Cancer Institute (Pittsburgh, PA), and Hoechst 33342 was purchased from Invitrogen (Carlsbad, CA). Dimethyl sulfoxide (DMSO; 99.9\% high-performance liquid chromatography grade, under argon) was from Alfa Aesar (Ward Hill, MA). Dulbecco's $\mathrm{Mg}^{2+}$ - and $\mathrm{Ca}^{2+}$-free phosphatebuffered saline (PBS) were purchased from Corning (Tewksbury, MA). The AlphaScreen Histidine (Nickel Chelate) Detection Kit, 500 assay points was purchased from Perkin Elmer (Waltham, MA), and Geneticin ${ }^{\mathrm{TM}}$ Selective Antibiotic (G418 Sulfate) pow- der was purchased from Fisher Scientific (Pittsburgh, PA). T4 DNA Ligase, BamHI (R0136S), and EcoRI (R3101S) were purchased from New England Biolabs (Ipswich, MA). FuGENE ${ }^{\mathrm{TM}} 6$ and FuGENE HD transfection Reagents were purchased from Promega (Madison, WI). Phusion High-Fideltiy DNA Polymerase (F530S) was purchased from Fisher Scientific. Bright$\mathrm{Glo}^{\mathrm{TM}}$ Luciferase Assay System was purchased from Promega. DHT [1,2,4,5,6,7-3H(N)]-(5 alpha-ANDROSTAN-17 beta-3-ol) was purchased from Perkin Elmer.

\section{Cell Lines and Tissue Culture}

PC3 cells were provided by the National Cancer Institute (NCI) as part of the NCI 60 tumor cell line panel. PC3 cells were maintained in RPMI 1640 (Gibco, Gaithersburg, MD) medium with $2 \mathrm{mM}$ L-glutamide (Invitrogen) that was supplemented with 10\% fetal bovine serum (FBS; Gemini Bio-Products, West Sacramento, CA), and $100 \mathrm{U} / \mathrm{mL}$ penicillin and streptomycin (Invitrogen).

PC3 cells that stably express AR-V7-GFP were kindly provided by Dr. Michael Mancini in the Departments of Molecular and Cellular Biology, and Pharmacology and Chemical Biology, Baylor College of Medicine, Houston, TX. PC3-AR-V7-GFP cells were maintained in Dulbecco's modified Eagle medium: nutrient mixture F-12 (DMEM/F12; Gibco) and supplemented with $10 \%$ FBS and $500 \mu \mathrm{g} / \mathrm{mL}$ geneticin (G418; Fisher Scientific).

We developed a PC3 cell line that stably expresses AR-FLGFP by transfecting PC3 cells with a pEGFP-C1-AR-FL expression plasmid kindly provided by Dr. Michael Mancini (Baylor College of Medicine). After transfection of PC3 cells with the pEGFP-C1-AR-FL expression plasmid, cells were placed under selection in culture media containing $600 \mu \mathrm{g} / \mathrm{mL}$ geneticin (G418; Fisher Scientific), and underwent three rounds of cell sorting for EGFP expression based on the EGFP mean fluorescent intensity measured and sorted on a BD FACSAria (BD Biosciences, San Jose, CA) performed by Dr. Hongmei Shen in the University of Pittsburgh Flow Cytometry Core Facility. The final PC3-AR-FL-GFP cell line was maintained in culture in RPMI 1640 (Gibco) media and supplemented with 10\% FBS (Gemini Bio-Products) and $600 \mu \mathrm{g} / \mathrm{mL}$ geneticin (G418; Fisher Scientific).

Human embryonic kidney 293 cells (HEK 293 cells; CRL-1537) were purchased from the American Type Culture Collection (Manassas, VA) and were maintained in DMEM (Cellgro10013CV; Corning) with $2 \mathrm{mM} \mathrm{L-glutamine} \mathrm{(Invitrogen)}$ that was supplemented with 10\% FBS (Gemini Bio-Products), and $100 \mathrm{U} / \mathrm{mL}$ penicillin and streptomycin (Invitrogen).

All cell lines were maintained in a humidified incubator at $37^{\circ} \mathrm{C}, 5 \% \mathrm{CO}_{2}$, and $95 \%$ humidity. 


\section{Compounds and Compound Handling}

To determine 50\% inhibition concentrations $\left(\mathrm{IC}_{50}\right)$ in each assay, 10-point twofold serial dilutions of test compounds in 100\% DMSO were performed by using a 384-well P30 dispensing head on the Janus MDT automated liquid handling platform (Perkin Elmer). Daughter plates containing $2 \mu \mathrm{L}$ of the serially diluted compounds in DMSO were prepared and replicated from the 384-well serial dilution master plates by using the Janus MDT platform that was outfitted with a 384well transfer head. Aluminum adhesive plate seals were applied, and plates were stored at $-20^{\circ} \mathrm{C}$. For testing in the bioassays, daughter plates were withdrawn from $-20^{\circ} \mathrm{C}$ storage, thawed to ambient temperature, and centrifuged for $1 \mathrm{~min}$ at $100 \mathrm{~g}$, and the plate seals were removed before the transfer of $38 \mu \mathrm{L}$ of serum-free media (SFM) into wells by using a Matrix pipettor (Thermo Fisher Scientific, Waltham, MA), to generate an intermediate stock concentration of validation compounds ranging from 0.977 to $500 \mu \mathrm{M}(5.0 \%$ DMSO). The diluted compounds were mixed by repeated aspiration and dispensation by using a 384-well P30 dispensing head on the Janus MDT platform and then, $5 \mu \mathrm{L}$ of diluted compounds was transferred to the wells of assay plates to provide a final concentration response ranging from 0.0977 to $50 \mu \mathrm{M}$ (0.5\% DMSO).

\section{AR-TIF2 PPIB Assay}

The AR-TIF2 PPIB HCS assay was performed in U-2 osteosarcoma cell line (U-2 OS) cells as described previously. ${ }^{22-25}$ Briefly, U-2 OS cells were coinfected with recombinant adenovirus biosensor expression constructs and seeded at 2,500 cells per well in 384-well collagen-coated microplates (No. 781956; Greiner Bio-One), and plates were incubated overnight at $37^{\circ} \mathrm{C}$ in $5 \% \mathrm{CO}_{2}$ and $95 \%$ humidity. To determine if compounds could block DHT-induced AR-TIF2 PPI formation, assay plates were preincubated with compounds for $3 \mathrm{~h}$ before the addition of $25 \mathrm{nM}$ DHT for $90 \mathrm{~min}$. To investigate whether compounds could disrupt pre-existing AR-TIF2 PPI complexes, assay plates were preincubated with $25 \mathrm{nM}$ DHT for $90 \mathrm{~min}$ before the transfer of compounds, and an additional 3 -h incubation. Maximum plate control wells $(n=32)$ in columns 1 and 2 were exposed to $25 \mathrm{nM}$ DHT and $\leq 0.25 \%$ DMSO, and minimum plate control wells $(n=32)$ in columns 23 and 24 were treated with $\leq 0.25 \%$ DMSO. Diluted compounds, DHT or DMSO $(5 \mu \mathrm{L})$, were transferred at the indicated concentrations as described above. After the appropriate incubation time with compounds and controls, assay plates were fixed by the addition of $50 \mu \mathrm{L}$ of prewarmed $\left(37^{\circ} \mathrm{C}\right) 7.4 \%$ formaldehyde and $2 \mu \mathrm{g} / \mathrm{mL}$ Hoechst 33342 in PBS and incubated at room temperature (RT) for $30 \mathrm{~min}$. Liquid was then aspirated, and plates were then washed twice with $85 \mu \mathrm{L}$ PBS, leaving the final wash in the plate. The plates were then sealed with adhesive aluminum plate seals. Fluorescent images of three fields of view in each of the DAPI (Hoechst stained nuclei), FITC (TIF2-GFP), and Texas Red (AR-RFP) channels were acquired on an ImageXpress Micro (IXM) automated HCS platform (Molecular Devices, LLC, Sunnyvale, CA) using a $10 \times$ Plan Fluor 0.3 NA objective. Images were then analyzed using the translocation enhanced (TE) image analysis module of the MetaXpress software as described previously. ${ }^{22-25}$

\section{TIF2 and SRC1 M2H Assays}

The 5xGAL4-TATA-luciferase reporter plasmid was a kind gift from Dr. Richard Maurer from the Oregon Health and Science University, ${ }^{50}$ and constructs pGAL4-hAR-658-919 (AR-LBD amino acids 658-919 expressed as a fusion protein with Gal4-DBD [galactose 4 DNA binding domain DNAbinding domain]), ${ }^{51} \mathrm{pVP} 16-S R C 1$ (full-length SRC1 expressed as a fusion with VP16 activation domain), ${ }^{52}$ and pVP16empty vector were kindly provided to us by Dr. Elizabeth Wilson, from UNC Chapel Hill. pVP16-TIF2 was generated by ligating TIF2, polymerase chain reaction (PCR) amplified from pCR-BluntII-TOPO-NCoA2 (TIF2) complementary DNA (cDNA; purchased from Dharmacon, Lafayette, CO), into the empty pVP16 vector.

HEK 293 cells were cotransfected with $5 \mathrm{ng}$ of pGal4-ARLBD, $10 \mathrm{ng}$ of either pVP16-TIF2 or pVP16-SRC1, and $20 \mathrm{ng}$ of the 5xGal4-TATA-Luc reporter. Cells were transiently bulk cotransfected with the three plasmids using the FuGENE 6 transfection reagent. Before the transfection of cells, each of the pGAL4-hAR-658-919, 5xGAL4-TATA-luciferase reporter, and either pVP16-TIF2 or pVP16-SRC1 plasmid DNAs were individually incubated with FuGENE 6 at a 3:1 ratio for $25 \mathrm{~min}$ at RT. Each FuGENE 6 to plasmid DNA mixture was then added to HEK 293 cells that were suspended in DMEM (Cellgro10013CV) with $2 \mathrm{mM}$ L-glutamine (Invitrogen) that was supplemented with 10\% FBS, and 5,000 cells in a volume of $40 \mu \mathrm{L}$ were seeded into the wells of 384well assay plates and cultured overnight at $37^{\circ} \mathrm{C}, 5 \% \mathrm{CO}_{2}$, and 95\% humidity. Twenty-four hours after cell seeding into assay plates, $5 \mu \mathrm{L}$ of serially diluted compounds were transferred to assay wells and plates were incubated at $37^{\circ} \mathrm{C}$, $5 \% \mathrm{CO}_{2}$, and $95 \%$ humidity for $3 \mathrm{~h}$ before $5 \mu \mathrm{L}$ of $0.25 \mu \mathrm{M}$ DHT ( $25 \mathrm{nM}$ final) was transferred into each well, and the assay plates were returned to the incubator for an additional $24 \mathrm{~h}$. After $24 \mathrm{~h}, 25 \mu \mathrm{L}$ of BrightGlo ${ }^{\circledR}$ reagent was added to the plate and the relative light units (RLUs) were captured on a SpectraMax M5e microtiter plate reader (Molecular Devices, LLC, San Jose, CA). 
PSA6.1 Promoter-Driven Luciferase Reporter Assay Developed in PC3-AR-FL-GFP Cells

The pPSA-6.1-Luc luciferase reporter plasmid was kindly provided by Dr. Zhou Wang in the Department of Urology of the University of Pittsburgh Hillman Cancer Institute. ${ }^{53}$ FuGENE HD and PSA6.1-Luc were combined at a 3:1 $(\mu \mathrm{L}: \mu \mathrm{g})$ ratio, respectively, in Opti-MEM and incubated for $25 \mathrm{~min}$ at RT before being used to bulk transfect AR-FL-EGFP-PC3 cells in RPMI 1640 media containing 1\% L-glutamine, and 10\% FBS. Transfected cells were then seeded into white opaque 384-well assay plates (No. 781080; Greiner Bio-One) at a seeding density of 3,000 cells per well in a volume of $40 \mu \mathrm{L}$ and incubated at $5 \% \mathrm{CO}_{2}, 37^{\circ} \mathrm{C}$, and $95 \%$ humidity for $24 \mathrm{~h}$. Each well received $20 \mathrm{ng}$ of PSA6.1 LUC plasmid DNA. After $24 \mathrm{~h}, 5 \mu \mathrm{L}$ of the serially diluted compounds was transferred to assay wells, and after a 3-h incubation, $5 \mu \mathrm{L}$ of $0.25 \mu \mathrm{M}$ DHT ( $25 \mathrm{nM}$ final) in SFM was transferred to each well and assay plates were returned to the incubator for an additional $24 \mathrm{~h}$ before $25 \mu \mathrm{L}$ of BrightGlo luciferase reagent (Promega) was added to the wells and the RLUs were captured on a SpectraMax M5e plate reader (Molecular Devices, LLC).

\section{AR-LBD::TIF2-Box III-LXXLL-Peptide Binding Assay}

The pET28a-AR-LBD (622-919) construct $^{54}$ was a gift from Dr. Fletterick and Dr. Nguyen of University of California San Francisco. Biotinylated-TIF2-box-III (738-756) peptide (Biotin-HN-CKKKENALLRYLLDKDDTKD-CONH ${ }_{2}$ ) and non-biotinylated TIF2-box-III (738-756) peptide $\left(\mathrm{H}_{2} \mathrm{~N}\right.$ CKKKENALLRYLLDKDDTKD- $\mathrm{CONH}_{2}$ ) were synthesized by the Peptide \& Peptoid Synthesis Facility, at the University of Pittsburgh Health Sciences Core Research Facilities. An ALPHAScreen kit containing streptavidin donor beads (SADB) and nickel chelate acceptor beads (Ni-AB) was purchased from Perkin Elmer. The assay was carried out in 384-well white opaque plates (No. 781080; Greiner Bio-One). One hundred fifty nanomolars of biotinylated TIF2-box III peptide was incubated with $5 \mu \mathrm{g} / \mu \mathrm{L}$ SA-BD for $30 \mathrm{~min}$ at RT in the dark. At the same time, $\mathrm{His}_{6}$-AR-LBD (400 ng/well) was incubated with $10 \mu \mathrm{M}$ DHT and $5 \mu \mathrm{g} / \mu \mathrm{L} \mathrm{Ni}^{2+}$-Abs for $30 \mathrm{~min}$ at RT in the dark. Around $18 \mu \mathrm{L}$ of the SA-DB bound biotinylated TIF2 peptide mixture was added to the assay plate before $5 \mu \mathrm{L}$ of the serially diluted compounds was transferred into assay wells and then $27 \mu \mathrm{L}$ of the $\mathrm{His}_{6}$-AR-LBD-Ni-DA bead mixture was added to the plate. Thirty-two wells containing 0.5\% DMSO were used as maximum controls and 32 wells containing a 500-fold excess of unlabeled TIF2-box-III $(75 \mu \mathrm{M})$ were used as minimum controls. The combined beadprotein-peptide-compound mixture was incubated for $1 \mathrm{~h}$ at
RT in the dark, and then, the RLUs were acquired at $520 \mathrm{~nm}$ after excitation at $680 \mathrm{~nm}$ on an Envision plate reader (Perkin Elmer).

\section{$\mathrm{H}^{3}$-DHT Radioligand Binding Assay}

The development of the $\mathrm{His}_{6}$-AR-LBD H ${ }^{3}$-DHT competition binding assay has been described previously. ${ }^{24}$ Briefly, the assay was carried out in 96-well $\mathrm{Cu}^{2+}$-coated plates (Thermo Fisher Scientific) that were incubated overnight at $4^{\circ} \mathrm{C}$ with $5 \mu \mathrm{g}$ per well $\mathrm{His}_{6}$-AR-LBD in $100 \mu \mathrm{L}$ of PBS. Unbound $\mathrm{His}_{6}{ }^{-}$ AR-LBD was aspirated, the plate was washed $3 \times$ with $100 \mu \mathrm{L}$ of $0.05 \%$ Tween 20 in PBS, and then blocked with $100 \mu \mathrm{L}$ of $1 \mathrm{mg} / \mathrm{mL}$ bovine serum albumin in PBS for $1 \mathrm{~h}$. After three more washes with $100 \mu \mathrm{L}$ of PBS and $0.05 \%$ Tween $20,40 \mu \mathrm{L}$ of PBS was added to the wells using a Matrix pipettor. Next, $5 \mu \mathrm{L}$ of diluted compound followed by $5 \mu \mathrm{L}$ of $100 \mathrm{nM} \mathrm{H}^{3}$-DHT were transferred into the wells using a Matrix pipettor. Compounds were tested in a 10-point twofold serial dilution series ranging between 0.098 and $50 \mu \mathrm{M}$ in the presence of $10 \mathrm{nM} \mathrm{H}^{3}$-DHT. After a 1-h coincubation, compounds and $\mathrm{H}^{3}$ DHT were aspirated and washed three times with $0.05 \%$ Tween 20 in PBS; $100 \mu \mathrm{L}$ of Microscint ${ }^{\mathrm{TM}}-20$ micro-scintillation cocktail buffer (Perkin Elmer) was added to each well, plates were sealed with adhesive plastic covers; and the counts per minute (CPMs) were captured in a TopCount NXT microtiter plate reader (Perkin Elmer).

\section{pGal4-AR-NTD Transactivation Assay}

The 5xGAL4-TATA-luciferase reporter plasmid was provided by Dr. Richard Maurer, ${ }^{50}$ as described above. AR NTD constructs pGAL4-hAR-1-503, AR amino acids 1-503 expressed as a fusion protein with Gal4-DBD, ${ }^{51}$ pVP16-SRC1 (full-length SRC1 expressed as a fusion with VP16 activation domain), ${ }^{52}$ and pVP16-empty vector were kindly provided to us by Dr. Elizabeth Wilson, from the University of North Carolina Chapel Hill. The pVP16-TIF2 construct was generated as described above. An empty pGALO vector was kindly provided by Dr. Gordon Tomaselli (Albert Einstein College of Medicine), and pGAL-hAR-1-558 and pGAL4AR3 (AR-V7) were generated by ligating PCR-amplified inserts into the pGALO M2H vector. PCR-amplified inserts were cut with BamHI and XbaI restriction enzymes. Primers were synthesized and purchased from Thermo Fisher Scientific.

HEK 293 cells were transiently bulk cotransfected with $5 \mathrm{ng}$ of the pGal4-AR-NTD (1-503) and $20 \mathrm{ng}$ of the $5 \times \mathrm{xGal} 4-$ TATA-Luc reporter plasmids using the FuGENE 6 transfection reagent (Promega). Before the cotransfection of cells, the pGAL4-hAR-1-503 and 5xGAL4-TATA-luciferase reporter 
plasmids were individually incubated with FuGENE 6 at a 3:1 ratio for $25 \mathrm{~min}$ at $\mathrm{RT}$ and the mixture was then added to HEK 293 cells that were suspended in DMEM (Cellgro10013CV) with $2 \mathrm{mM}$ L-glutamine (Invitrogen), which was supplemented with 10\% FBS (Gemini Bio-Products). Five thousand cells in a volume of $40 \mu \mathrm{L}$ were then seeded into the wells of 384-well assay plates and cultured overnight at $37^{\circ} \mathrm{C}, 5 \%$ $\mathrm{CO}_{2}$, and 95\% humidity. Twenty-four hours after cell seeding, $10 \mu \mathrm{L}$ of serially diluted compounds was transferred to the wells and assay plates were returned to the incubator for an additional $24 \mathrm{~h}$ before $25 \mu \mathrm{L}$ of BrightGlo reagent was added to the plate wells, and the RLUs were captured on a SpectraMax M5e microtiter plate reader.

\section{PSA6.1 Promoter-Driven Luciferase Reporter}

Assay in PC3-AR-V7-GFP Cells

PC3-AR-V7-GFP cells were transfected with a mixture of FuGENE HD and the PSA-6.1-Luc reporter plasmid combined at a 3:1 ( $\mu \mathrm{L}: \mu \mathrm{g})$ ratio in Opti-MEM (Gibco), which had been incubated for $25 \mathrm{~min}$ at RT before being added to cells that were suspended in RPMI 1640 (Gibco) media containing 1\% Lglutamine (Invitrogen) and 10\% FBS (Gemini Bio-Products). Bulk transfected PC3-AR-V7-GFP cells were then seeded into white opaque 384-well assay plates (No. 781080; Greiner BioOne) at a seeding density of 3,000 cells per well in a volume of $40 \mu \mathrm{L}$ and incubated at $5 \% \mathrm{CO}_{2}, 37^{\circ} \mathrm{C}$, and $95 \%$ humidity for $24 \mathrm{~h}$. Each well received $20 \mathrm{ng}$ of PSA-6.1 LUC plasmid DNA. After $24 \mathrm{~h}, 5 \mu \mathrm{L}$ of the serially diluted compounds were transferred to assay wells and the plates were returned to the incubator for an additional $24 \mathrm{~h}$ before $25 \mu \mathrm{L}$ of BrightGlo luciferase reagent (Promega) was added to the wells, and the RLUs were captured on a SpectraMax M5e plate reader (Molecular Devices, LLC).

\section{UBE2C Promoter-Driven Luciferase Reporter}

Assay in PC3-AR-V7-GFP Cells

The pGL4.28-UBE2C 20bpX3 luciferase reporter plasmid ${ }^{55}$ was kindly provided by Dr. Yan Dong from Tulane University. FuGENE HD and the UBE2C-Luc plasmid were combined at a $3: 1(\mu \mathrm{L}: \mu \mathrm{g})$ ratio in Opti-MEM and incubated for $25 \mathrm{~min}$ at RT before being added to PC3-AR-V7-GFP cells that were suspended in RPMI 1640 (Gibco) media containing 1\% L-glutamine (Invitrogen) and 10\% FBS (Gemini Bio-Products). Bulk transfected PC3-AR-V7-GFP cells were then seeded into white opaque 384-well assay plates (No. 781080; Greiner BioOne) at a seeding density of 3,000 cell per well in a volume of $40 \mu \mathrm{L}$ and incubated at $5 \% \mathrm{CO}_{2}, 37^{\circ} \mathrm{C}$, and $95 \%$ humidity for $24 \mathrm{~h}$. Each well received $10 \mathrm{ng}$ of UBE2C-LUC plasmid DNA. After $24 \mathrm{~h}, 10 \mu \mathrm{L}$ of the serially diluted compounds were transferred to assay wells and the plates were returned to the incubator for an additional $24 \mathrm{~h}$ before $25 \mu \mathrm{L}$ of BrightGlo luciferase reagent (Promega) was added to the wells, and the RLUs were captured on a SpectraMax M5e plate reader (Molecular Devices, LLC).

\section{RESULTS}

\section{How Did the Test Compounds Perform} in the AR-TIF2 PPIB HCS Assay?

We first wanted to benchmark how the selected test compounds would perform in the AR-TIF2 HCS biosensor assay (Fig. 1, Supplementary Figs. S1 and S2, and Table 1). In two independent assay runs, the DHT-induced AR-TIF2 PPI formation ( $C$ then $\mathrm{D}$ ) format produced $\mathrm{Z}$-factor coefficients $\geq 0.53$ and signal-to-background ( $\mathrm{S}: \mathrm{B})$ ratios $\geq 9.5$, and the format designed to identify disruptors of pre-existing AR-TIF2 PPIs (D then C) produced Z-factor coefficients $\geq 0.51$ and S:B ratios $\geq 21.7$ (Supplementary Table S1). As expected, 3-h preexposure to AR antagonists inhibited DHT-induced AR-TIF2 PPI formation in a concentration-dependent manner, with flutamide, bicalutamide, and enzalutamide exhibiting $\mathrm{IC}_{50} \mathrm{~S}$ of $8.77 \pm 1.59,2.49 \pm 0.20$, and $1.44 \pm 0.16 \mu \mathrm{M}$, respectively (Fig. 1A and Table 1). Interestingly, flutamide, bicalutamide, and enzalutamide also disrupted pre-existing AR-TIF2 PPI complexes with $\mathrm{IC}_{50}$ s of $20.6 \pm 4.46,4.01 \pm 1.37$, and $2.71 \pm$ $0.27 \mu \mathrm{M}$, respectively (Fig. $1 D$ and Table 1). Consistent with our previous observations, the Hsp90 inhibitor 17-AAG produced an $\mathrm{IC}_{50}$ of $0.20 \pm 0.01 \mu \mathrm{M}$ in the AR-TIF2 PPI formation format, and an $\mathrm{IC}_{50}$ of $0.39 \pm 0.01 \mu \mathrm{M}$ in the disruption of preexisting AR-TIF2 PPIs format (Fig. 1C, F, and Table 1). In contrast, neither of the $\mathrm{NH}_{2-}$ terminus acting compounds nor the Cyp17a1 inhibitor abiraterone acetate produced calculable $\mathrm{IC}_{50} \mathrm{~S}$ in either format of the AR-TIF2 PPIB assay at $\leq 50 \mu \mathrm{M}$ (Fig. 1B, C, E, F, and Table 1). None of the test compounds significantly reduced the number of U-2 OS cells (Hoechststained nuclei) detected in DAPI channel images, indicating that during a 3-h exposure period, they were not acutely cytotoxic at the concentrations tested.

\section{Do Test Compounds Inhibit the TIF2 or SRC1 AR-LBD M2H Assays?}

We developed $\mathrm{M} 2 \mathrm{H}$ assays to measure the interactions between the AF-2 surface formed by DHT binding to the AR-LBD with two coactivators, either TIF2 or SRC1. The M2H assays were developed in the HEK 293 cell background transiently transfected with either full-length TIF2 or SRC1 chimeras attached to the VP16 transcriptional activation domain, ARLBD fused to the Gal4 DNA binding domain, and the 5xGal4TATA-Luc reporter construct (Supplementary Fig. S3A). To 


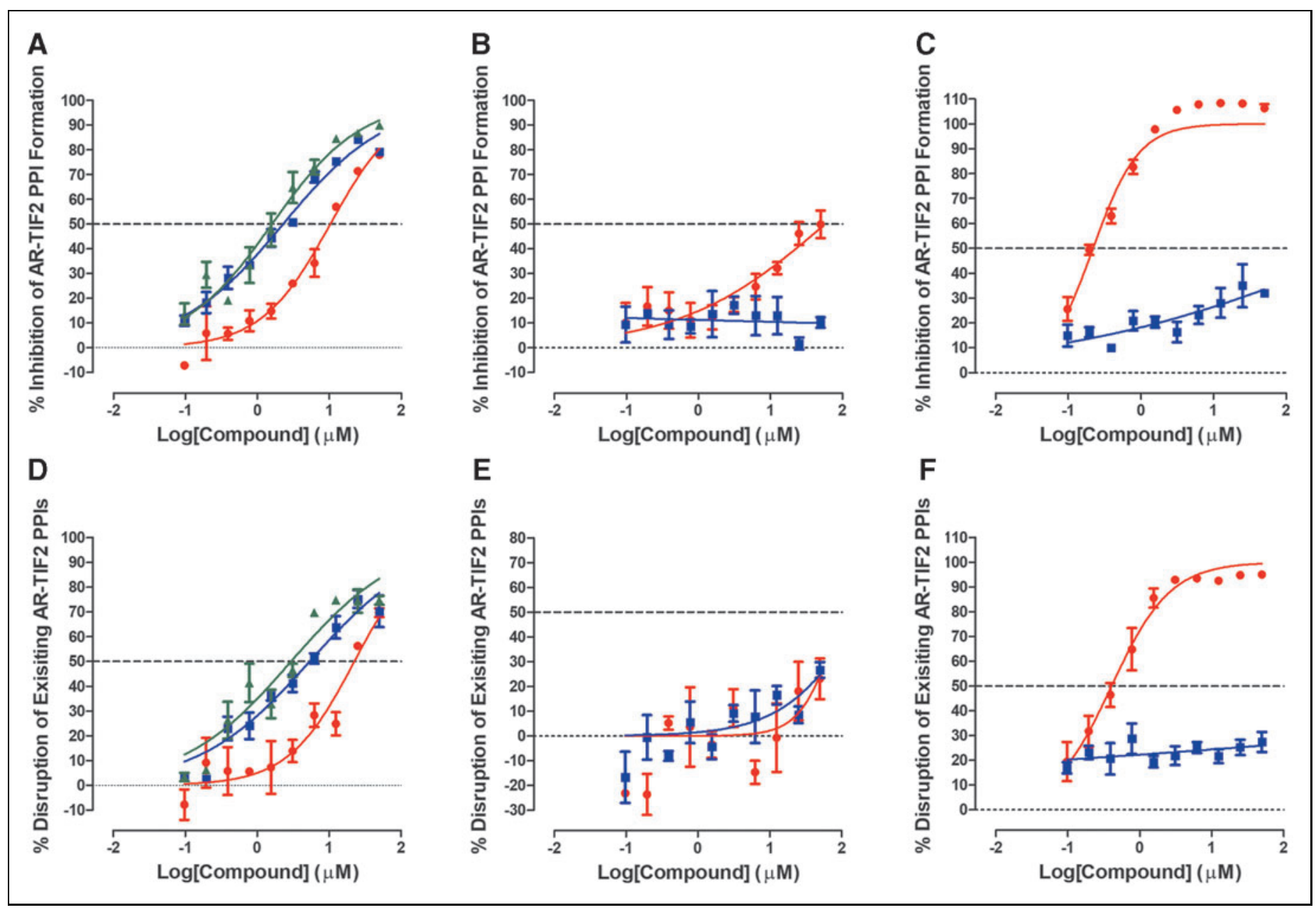

Fig. 1. Inhibition of DHT-induced AR-TIF2 PPI formation (A-C) or disruption of preformed AR-TIF2 PPI complexes (D-F). U-2 OS cells were coinfected with AR-RFP and TIF2-GFP rAV biosensors, and 3,000 cells were seeded into the wells of 384-well assay plates and cultured overnight at $37^{\circ} \mathrm{C}, 5 \% \mathrm{CO}_{2}$, and $95 \%$ humidity. After $24 \mathrm{~h}$, test compounds and DHT were added to cells in two formats. To determine if test compounds could inhibit DHT-induced AR-TIF2 PPI formation (A-C), coinfected U-2 OS cells were exposed to compounds at the indicated concentrations for $3 \mathrm{~h}$ before treatment with $25 \mathrm{nM} \mathrm{DHT}$ for $90 \mathrm{~min}$; (A) (red-filled circle) flutamide, (blue-filled square) bicalutamide, and (green-filled triangle) enzalutamide, (B) (red-filled circle) compound No. 10, (blue-filled square) EPI-oo1, (C) (red-filled circle) 17-AAG, and (blue-filled square) abiraterone acetate, To determine if test compounds could disrupt preformed AR-TIF2 PPI complexes (D-F), coinfected U-2 OS cells were exposed to $25 \mathrm{nM}$ of DHT for 90 min before treatment with the indicated concentrations of compounds for $3 \mathrm{~h}$; (D) (red-filled circle) flutamide, (blue-filled square) bicalutamide, and (green-filled triangle) enzalutamide, (E) (red-filled circle) compound No. 10, (blue-filled square) EPI-001, (F) (red-filled circle) 17-AAG, and (blue-filled square) abiraterone acetate. The AR-TIF2 PPI data were normalized to maximum (25 nM DHT $+0.5 \%$ DMSO, $n=32)$ and minimum (0.5\% DMSO, $n=32)$ plate controls, and the percent inhibition was plotted as the mean \pm SD $(n=3)$ values from triplicate wells for each compound concentration. Representative experimental data from one of two independent experiments are shown. 17-AAG, 17-allylaminogeldanamycin; AR, androgen receptor; DHT, dihydrotestosterone; DMSO, dimethyl sulfoxide; GFP, green fluorescent protein; PPI, protein-protein interaction; rAV, recombinant adenovirus; RFP, red fluorescent protein; SD, standard deviation; TIF2, transcriptional intermediary factor 2; U-2 OS, U-2 osteosarcoma cell line.

determine the optimal amounts of pGal4-AR-LBD, pVP16TIF2, or pVP16-SRC1 plasmids, we performed cross-titration transient transfection experiments with increasing amounts of the pGal4-AR-LBD and pVP16-coactivator plasmids at a fixed amount $(20 \mathrm{ng}$ ) of 5xGal4-TATA-Luc reporter construct (Supplementary Fig. S3B, E). Only HEK 293 cells that were cotransfected with all three plasmids and were exposed to DHT exhibited a luciferase response substantially greater than background. In both the TIF2 and SRC1 M2H assays, the DHTinduced luciferase response increased in a linear manner as the amount of pGal4-AR-LBD cotransfected into the HEK 293 cells increased. Similarly, the DHT-induced M2H luciferase 
Table 1. Test Compound $\mathbf{5 0} \%$ Inhibition Concentration Summary in AR-TIF2 Protein-Protein Interaction Biosensor

\begin{tabular}{l|c|c|c|c|c|c}
\multirow{2}{*}{ Test compound } & \multicolumn{5}{c}{ AR-TIF2 PPIB IC $50(\mu \mathrm{M})$} \\
\cline { 2 - 7 } & \multicolumn{2}{|c|}{ C then $\mathbf{D}^{\mathbf{a}}$} & \multicolumn{2}{c}{ D then C } & U-2 OS Cytox \\
\cline { 2 - 7 } Flutamide & 8.77 & 1.59 & 20.6 & 4.46 & $>50$ & N/A \\
\hline Bicalutamide & 2.49 & 0.2 & 4.01 & 1.37 & $>50$ & N/A \\
\hline Enzalutamide & 1.44 & 0.16 & 2.71 & 0.27 & $>50$ & N/A \\
\hline Compound-10 & $>50$ & N/A & $>50$ & N/A & $>50$ & N/A \\
\hline EPI-001 & $>50$ & N/A & $>50$ & N/A & $>50$ & N/A \\
\hline 17-AAG & 0.2 & 0.02 & 0.39 & 0.01 & $>50$ & N/A \\
\hline Abiraterone & $>50$ & N/A & $>50$ & N/A & $>50$ & N/A \\
\hline
\end{tabular}

${ }^{\text {aP }}$ retreatment with compound before DHT addition.

${ }^{b}$ Pretreatment with DHT before compound addition.

17-AAG, 17-allylaminogeldanamycin; AR, androgen receptor; Cytox, cytotoxicity; DHT, dihydrotestosterone; $I C_{50}, 50 \%$ inhibition concentration; N/A, not applicable; PPIB, protein-protein interaction biosensor; SD, standard deviation; TIF2, transcriptional intermediary factor 2; U-2 OS, U-2 osteosarcoma cell line.

response increased in a roughly linear manner as the amount of the pVP16-coactivator constructs cotransfected into the HEK 293 cells increased. The magnitude of the TIF2 M2H response was 30-fold higher than the signal in the SRC1 M2H assay (Supplementary Fig. S3B versus E). The final amounts of plasmid DNAs selected for the optimized $\mathrm{M} 2 \mathrm{H}$ assays were $5 \mathrm{ng}$ for pGal-AR-LBD, $10 \mathrm{ng}$ each of the pVP16-coactivator constructs, and $20 \mathrm{ng}$ of the $5 \mathrm{xGal4}$-TATA-Luc reporter construct. A DHT concentration response was performed to determine the optimal concentration of DHT to use in subsequent $\mathrm{M} 2 \mathrm{H}$ experiments. DHT exhibited $\mathrm{EC}_{50} \mathrm{~s}$ of 0.619 and $2.23 \mathrm{nM}$ for the TIF2 and SRC1 M2H assays, respectively (Supplementary Fig. S3C, F), and we selected $25 \mathrm{nM}$ DHT as a suitable agonist concentration for activating both $\mathrm{M} 2 \mathrm{H}$ assay formats. DMSO tolerance experiments in the TIF2 and SRC1 $\mathrm{M} 2 \mathrm{H}$ formats indicated that $0.5 \%$ DMSO would not interfere in either assay (Supplementary Fig. S3D, G).

Both $\mathrm{M} 2 \mathrm{H}$ assays performed well in two independent compound testing runs with the SRC1 AR-LBD assay producing $\mathrm{Z}$-factor coefficients $\geq 0.47$ and $\mathrm{S}: \mathrm{B}$ ratios $\geq 10.4$, and the TIF2 AR-LBD assay producing Z-factor coefficients $\geq 0.48$ and S:B ratios $\geq 45.6$ (Supplementary Table S1). All seven test compounds inhibited DHT-induced AR-LBD interactions with the TIF2 and SRC1 coactivators in a concentration-dependent manner (Figs. 2 and 3). In the TIF2 M2H assay, the anti- androgens flutamide, bicalutamide, and enzalutamide exhibited $\mathrm{IC}_{50} \mathrm{~S}$ of $3.01 \pm 0.75,2.01 \pm 0.99$, and $2.36 \pm 1.44 \mu \mathrm{M}$, respectively, and the Hsp90 inhibitor 17-AAG produced an $\mathrm{IC}_{50}$ of $0.33 \pm 0.02 \mu \mathrm{M}$ (Fig. $2 \mathrm{~A}$ and Table 2). In the SRC1 M2H assay, flutamide, bicalutamide, and enzalutamide exhibited $\mathrm{IC}_{50} \mathrm{~S}$ of $2.53 \pm 0.66,1.49 \pm 0.69$, and $1.37 \pm 0.91 \mu \mathrm{M}$, respectively (Fig. $3 A$ and Table 2), and 17-AAG produced an $\mathrm{IC}_{50}$ of $0.45 \pm 0.08 \mu \mathrm{M}$ (Fig. 3C and Table 2). None of these four test compounds produced substantial levels of cytotoxicity in either M2H assay (Figs. 2D, 2F, 3D, 3F, and Table 2). Although compound No. 10, EPI-001, and abiraterone acetate also inhibited both $\mathrm{M} 2 \mathrm{H}$ assays, they likewise displayed substantial concentration-dependent cytotoxicity in HEK 293 cells (Figs. 2B, 2C, 2E, 2F, 3B, 3C, 3E, 3F, and Table 2). Indeed, compound-induced cytotoxicity may be the primary mechanism of decreasing the $\mathrm{M} 2 \mathrm{H}$ reporter signals by compound No. 10, EPI-001, and abiraterone acetate (Figs. 2E, 2F, 3E, 3F, and Table 2). Overall, none of the test compounds were selective at disrupting DHT-induced AR-LBD interactions with either TIF2 or SRC1.

Do Test Compounds Inhibit the PSA6.1 Promoter-Driven Luciferase Reporter Assay Conducted in PC3 Cells Stably Expressing AR-FL-EGFP?

We wanted to examine whether test compounds could block the transcriptional activity of AR-FL expressed in a PCa cell background. The PSA6.1 luciferase reporter is controlled by a fragment of the PSA promoter that contains three AREs fused to a luciferase reporter gene. ${ }^{53}$ First, we generated a cell line that stably expresses AR-FL-EGFP in the AR null PC3 PCa cell line. Supplementary Figure S4A shows representative grayscale DAPI and FITC channel and color composite images of the AR-FL-EGFP-PC3 cell line \pm DHT exposure, which were stained with the Hoechst DNA stain. For comparison, images of the AR null parental PC 3 cell line are also presented. Exposure to DHT alters the subcellular localization of the AR-FL-EGFP to a predominantly nuclear distribution in images captured on the IXM HCS platform (Supplementary Fig. S4A). Exposure of AR-FL-EGFP-PC3 cells to DHT increased the inner/outer intensity ratio output of the TE image analysis module, which is a measure of the mean average fluorescence intensity of AR-FL-GFP in the nucleus, divided by the mean average fluorescence intensity of AR-FL-GFP in the cytoplasm. In PC3-AR-FL-EGFP cells, DHT induced a concentration-dependent increase in the inner/outer intensity ratio with an $\mathrm{EC}_{50}$ of $1.23 \pm 0.131 \mathrm{nM}$ (Supplementary Fig. S4B). After PC3-AR-FL-EGFP cells were transiently transfected with the PSA6.1-Luc reporter construct, DHT induced a concentration-dependent increase in 

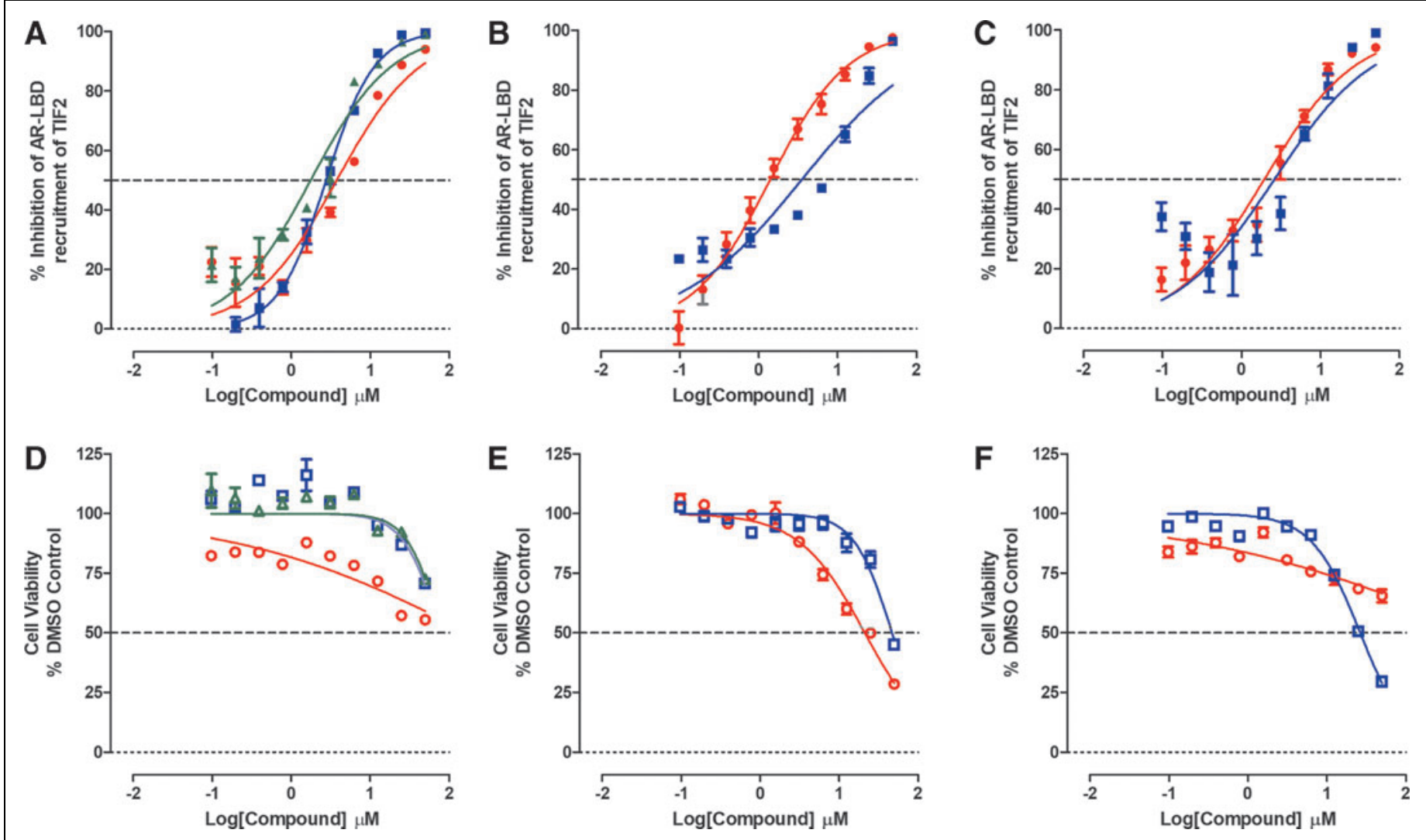

Fig. 2. Inhibition of DHT-induced $M 2 \mathrm{H}$ Interactions between AR-LBD and TIF2. HEK 293 cells that were cotransfected with three plasmid constructs, pGAL4-AR-LBD, 5xGal4-TATA-Luc, and pVP16-TIF2, were seeded at 5,000 cells per well in 384-well assay plates, and cultured overnight at $37^{\circ} \mathrm{C}, 5 \% \mathrm{CO}_{2}$, and $95 \%$ humidity. After $24 \mathrm{~h}$, cells were exposed to test compounds at the indicated concentrations for $3 \mathrm{~h}$ before the addition of $25 \mathrm{nM}$ DHT. After an additional 24 -h incubation, BrightGlo ${ }^{\circledR}$ reagent was added to the assay plates and the RLUs were captured on a SpectraMax M5e microtiter plate reader. The M2H RLU's data were normalized to maximum (25 $\mathrm{nM}$ DHT +0.5\% DMSO, $n=32)$ and minimum ( $0.5 \%$ DMSO, $n=32)$ plate controls, and the percent inhibition was plotted as the mean \pm SD $(n=3)$ values from triplicate wells for each compound concentration. The normalized percent inhibition of the DHT-induced AR-LBD TIF2 M2H responses for (A) (red-filled circle) flutamide, (blue-filled square) bicalutamide, and (green-filled triangle) enzalutamide, (B) (red-filled circle) compound No. 10, (blue-filled square) EPI-001, (C) (red-filled circle) 17-AAG, and (blue-filled square) abiraterone acetate are presented. The corresponding cell viability data for compound-treated wells were normalized to $0.5 \%$ DMSO control wells $(n=32)$, and the percent cell viability plotted as the mean \pm SD $(n=3)$ values from triplicate wells for each compound concentration is presented in (D) (red open circle) flutamide, (blue open square) bicalutamide, and (green open triangle) enzalutamide, (E) (red open circle) compound No. 10, (blue open square) EPI-o01, (F) (red open circle) 17-AAG, and (blue open square) abiraterone acetate. Representative experimental data from one of two independent experiments are shown. LBD, ligand-binding domain; Luc, luciferase; $\mathrm{M} 2 \mathrm{H}$, mammalian 2-hybrid; pGal4, DNA-binding domain of the Gal4 protein derived from yeast; RLUs, relative light units.

reporter activity with an $\mathrm{EC}_{50}$ of $0.280 \pm 0.034 \mathrm{nM}$ (Supplementary Fig. S4C). We selected $25 \mathrm{nM}$ DHT as a suitable agonist concentration for conducting the PSA6.1-Luc reporter assay in the PC3-AR-FL-EGFP cell background.

In independent compound testing runs in the PC3-AR-FLGFP-PSA-6.1-Luc reporter assay, the assay performed well with Z-factor coefficients $\geq 0.40$ and S:B ratios $\geq 5.6$ (Supplementary Table S1). As anticipated, the antiandrogens flutamide, bicalutamide, and enzalutamide inhibited DHTinduced reporter activity in a concentration-dependent manner, exhibiting $\mathrm{IC}_{50} \mathrm{~S}$ of $17.4 \pm 3.91,21.9 \pm 4.80$, and $19.5 \pm 3.45 \mu \mathrm{M}$, respectively (Fig. $4 B$ and Table 2). Similarly, the Hsp90 inhibitor 17-AAG produced an $\mathrm{IC}_{50}$ of $1.72 \pm$ $0.48 \mu \mathrm{M}$ (Fig. $4 \mathrm{D}$ and Table 2). In contrast, compound No. 10, EPI-001 and abiraterone acetate failed to inhibit DHTinduced transcriptional activity in PC3-AR-FL-EGFP cells at $\leq 50 \mu \mathrm{M}$ (Fig. 4 C, D, and Table 2). None of the test compounds exhibited evidence of cytotoxicity in PC3-AR-FL-EGFP cell line viability assays conducted in parallel with the reporter assays (Table 2). 

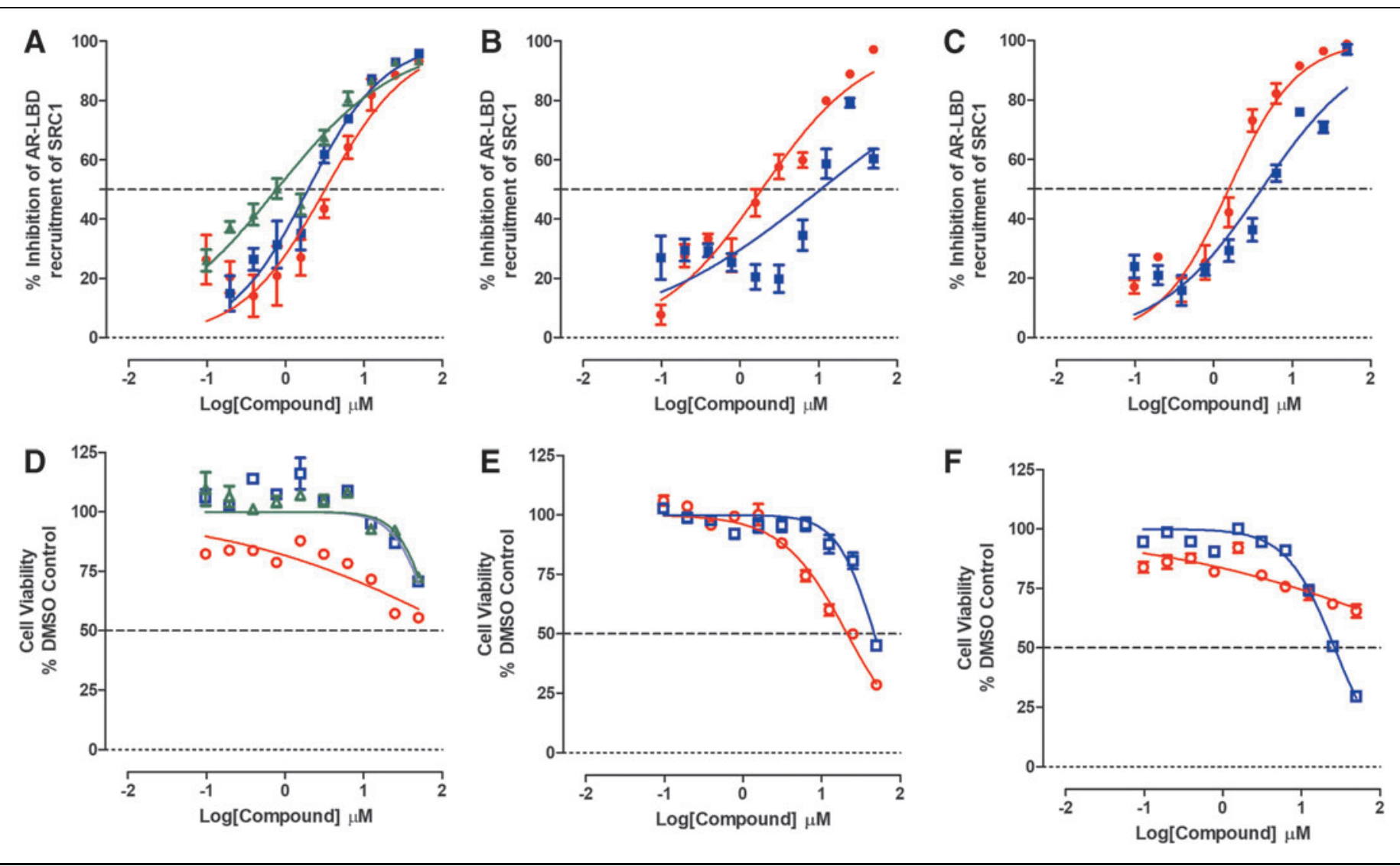

Fig. 3. Inhibition of DHT-induced $\mathrm{M}_{2} \mathrm{H}$ Interactions between AR-LBD and SRC1. HEK 293 cells that were cotransfected with three

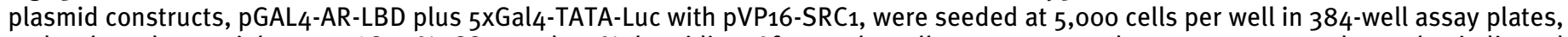
and cultured overnight at $37^{\circ} \mathrm{C}, 5 \% \mathrm{CO}_{2}$, and $95 \%$ humidity. After $24 \mathrm{~h}$, cells were exposed to test compounds at the indicated concentrations for $3 \mathrm{~h}$ before the addition of $25 \mathrm{nM} \mathrm{DHT}$. After an additional $24^{-\mathrm{h}}$ incubation, BrightGlo reagent was added to the assay plates and the RLUs were captured on a SpectraMax $M_{5} \mathrm{e}$ microtiter plate reader. The M2H RLU's data were normalized to maximum (25 nM DHT +0.5\% DMSO, $n=32)$ and minimum ( $0.5 \%$ DMSO, $n=32)$ plate controls, and the percent inhibition was plotted as the mean \pm SD $(n=3)$ values from triplicate wells for each compound concentration. The normalized percent inhibition of the DHTinduced AR-LBD SRC1 $\mathrm{M}_{2} \mathrm{H}$ responses for (A) (red-filled circle) flutamide, (blue-filled square) bicalutamide, and (green-filled triangle) enzalutamide, (B) (red-filled circle) compound No. 10, (blue-filled square) EPI-001, (C) (red-filled circle) 17-AAG, and (blue-filled square) abiraterone acetate is presented. The corresponding cell viability data for compound-treated wells were normalized to $0.5 \%$ DMSO control wells $(n=32)$, and the percent cell viability plotted as the mean \pm SD $(n=3)$ values from triplicate wells for each compound concentration is presented in (D) (red open circle) flutamide, (blue open square) bicalutamide, and (green open triangle) enzalutamide, (E) (red open circle) compound No. 10, (blue open square) EPI-001, (F) (red open circle) 17-AAG, and (blue open square) abiraterone acetate. Representative experimental data from one of two independent experiments are shown. SRC, steroid receptor coactivator.

Do Test Compounds Antagonize $\mathrm{H}^{3}$-DHT

Binding to the AR-LBD?

The $\mathrm{H}^{3}$-DHT radioligand binding assay was incorporated into our screening paradigm to identify and deprioritize hits from the AR-TIF2 biosensor HCS assay that inhibited/disrupted AR-TIF2 PPIs through ligand binding antagonism. This assay specifically measures the displacement of $\mathrm{H}^{3}$-DHT from AR-LBD in the presence of increasing concentrations of test compounds and was performed as described previously. ${ }^{24}$ The $\mathrm{H}^{3}$-DHT radioligand binding assay performed well in two independent compound testing runs and produced Z-factor coefficients $\geq 0.79$ and S:B ratios $\geq 38.0$ (Supplementary Table S1). As expected, the AR antagonists flutamide, bicalutamide, and enzalutamide displaced $\mathrm{H}^{3}$-DHT binding to $\mathrm{His}_{6}$-AR-LBD with $\mathrm{IC}_{50}$ values of $48.6 \pm 19.5,5.53 \pm 3.27$, and $50.2 \pm 14.4 \mu \mathrm{M}$, respectively (Fig. $5 \mathrm{~A}$ and Table 2). Since none of the other test compounds interact with the AR ligand binding site, it was reassuring that they failed to displace $\mathrm{H}^{3}$-DHT binding to $\mathrm{His}_{6}$-AR-LBD at $\leq 50 \mu \mathrm{M}$ (Fig. 5B, C, and Table 2). 


\begin{tabular}{|c|c|c|c|c|c|c|c|c|c|c|c|c|c|c|}
\hline \multirow{3}{*}{$\begin{array}{l}\text { Assays } \\
\text { Test compound }\end{array}$} & \multicolumn{6}{|c|}{$\begin{array}{c}\text { Mammalian 2-hybrid } \\
\qquad \mathrm{IC}_{50}(\mu \mathrm{M})\end{array}$} & \multicolumn{4}{|c|}{$\begin{array}{c}\text { PSA6.1-Luc reporter } \\
\text { IC }_{50}(\mu \mathrm{M})\end{array}$} & \multirow{2}{*}{\multicolumn{2}{|c|}{$\begin{array}{c}\text { LXXLL-AR-LBD } \\
\text { binding IC } \text { Fo }_{50}(\mu \mathrm{M})\end{array}$}} & \multirow{2}{*}{\multicolumn{2}{|c|}{$\begin{array}{c}\mathrm{H}^{3}-\mathrm{DHT} \text { binding } \\
\mathrm{IC}_{50}(\mu \mathrm{M}) \\
\text { AR-LBD }\end{array}$}} \\
\hline & \multicolumn{2}{|c|}{ TIF2 } & \multicolumn{2}{|c|}{ SRC1 } & \multicolumn{2}{|c|}{ HEK 293 cell Cytox } & \multicolumn{2}{|c|}{ PC3-AR-GFP } & \multicolumn{2}{|c|}{ Cytox } & & & & \\
\hline & Mean & SD & Mean & SD & Mean & SD & Mean & SD & Mean & SD & Mean & SD & Mean & SD \\
\hline Flutamide & 3.01 & 0.75 & 2.53 & 0.66 & $>50$ & $\mathrm{~N} / \mathrm{A}$ & 17.4 & 3.91 & $>50$ & N/A & $>50$ & $\mathrm{~N} / \mathrm{A}$ & 48.6 & 19.5 \\
\hline Bicalutamide & 2.01 & 0.99 & 1.49 & 0.69 & $>50$ & $\mathrm{~N} / \mathrm{A}$ & 21.9 & 4.8 & $>50$ & N/A & $>50$ & $\mathrm{~N} / \mathrm{A}$ & 5.53 & 3.27 \\
\hline Enzalutamide & 2.36 & 1.44 & 1.37 & 0.91 & $>50$ & $\mathrm{~N} / \mathrm{A}$ & 19.5 & 3.45 & $>50$ & N/A & $>50$ & $\mathrm{~N} / \mathrm{A}$ & 50.2 & 14.4 \\
\hline Compound-10 & 2.32 & 0.91 & 1.99 & 0.19 & 26.5 & 10.9 & $>50$ & $N / A$ & $>50$ & N/A & $>50$ & $\mathrm{~N} / \mathrm{A}$ & $>50$ & $\mathrm{~N} / \mathrm{A}$ \\
\hline EPI-001 & 9.15 & 2.47 & 8.15 & 1.27 & 36.6 & 9.3 & $>50$ & $N / A$ & $>50$ & N/A & $>50$ & $\mathrm{~N} / \mathrm{A}$ & $>50$ & $\mathrm{~N} / \mathrm{A}$ \\
\hline 17-AAG & 0.33 & 0.02 & 0.45 & 0.08 & $>50$ & $\mathrm{~N} / \mathrm{A}$ & 1.72 & 0.48 & $>50$ & N/A & 29.8 & 9.78 & $>50$ & $\mathrm{~N} / \mathrm{A}$ \\
\hline Abiraterone & 4.47 & 1.96 & 2.43 & 0.64 & 30.5 & 7.72 & $>50$ & $N / A$ & $>50$ & N/A & $>50$ & $\mathrm{~N} / \mathrm{A}$ & $>50$ & $\mathrm{~N} / \mathrm{A}$ \\
\hline
\end{tabular}

AR-LBD, ligand-binding domain of the androgen receptor; GFP, green fluorescent protein; $H^{3}$-DHT, tritiated dihydrotestosterone; HEK 293 cells, human embryonic kidney 293 cells; Luc, luciferase; LXXLL, coactivator leucine rich binding motifs; PC3, human prostate cancer cell line; PSA, prostate-specific antigen; SRC1, steroid receptor coactivator 1.

Do Test Compounds Inhibit TIF2 Box III LXXLL

Peptide Binding to the AR-LBD?

We developed a biochemical assay to measure the binding interactions between the AR AF-2 surface formed by the DHT-activated AR-LBD and a peptide containing the LXXLL motif located in box III of the TIF2 coactivator's nuclear interaction domain (NID). It has been reported that the LXXLL motif from box III of the TIF2 NID contributes most of the binding interactions with the AR AF-2 surface. ${ }^{37,39,56,57}$ In our assay, the histidine-tagged AR-LBD protein was chelated to $\mathrm{Ni}^{2+}$-coated acceptor ALPHAScreen beads, and the biotinylated TIF2 Box III LXXLL peptide was bound to SA-DB. Binding of the two interacting partners promotes proximity between the donor and acceptor beads, which can be quantified through increased light emission at a wavelength of $520-620 \mathrm{~nm}$ after excitation at $680 \mathrm{~nm}^{58}$ (Supplementary Fig. S5A). To determine the optimal amounts of the $\mathrm{His}_{6}$-AR-LBD protein and biotin-TIF2-LXXLL-peptide to use in the ALPHAScreen assay, we performed titration experiments (Supplementary Fig. S5B, C). Based on the data from these experiments, we selected $400 \mathrm{ng} /$ well of the $\mathrm{His}_{6}{ }^{-}$ AR-LBD protein and $150 \mathrm{nM}$ biotin-TIF2-LXXLL-peptide for the optimized ALPHAScreen assay conditions. Under these conditions, the apparent $\mathrm{Kd}$ for the biotin-TIF2-LXXLLpeptide binding to $\mathrm{His}_{6}$-AR-LBD was $151.3 \mathrm{nM}$, and the Bmax ALPHAScreen signal was extrapolated at 42,880 CPMs (Supplementary Fig. S5C).
In competitive displacement binding experiments, nonbiotinylated TIF2-LXXLL-peptide exhibited an $\mathrm{IC}_{50}$ of $2.76 \mathrm{nM}$ for the displacement of biotin-TIF2-LXXLL-peptide binding from $\mathrm{His}_{6}$-AR-LBD (Fig. 6A). The biotin-TIF2-LXXLL-peptide:: AR-LBD ALPHAScreen assay performed well in two independent test compound runs and produced Z-factor coefficients $\geq 0.74$ and S:B ratios $\geq 33.4$ (Supplementary Table S1). We did not anticipate that any of the test compounds would inhibit or disrupt LXXLL motif binding to the AF-2 surface because they either bind to the ligand binding site or NTD of AR, or to other protein targets (Hsp90 or Cyp17a1). Surprisingly, the Hsp90 inhibitor 17-AAG exhibited an $\mathrm{IC}_{50}$ of $29.8 \pm 9.78 \mu \mathrm{M}$ in the biotin-TIF2-LXXLL-peptide::AR-LBD binding assay, and although the antiandrogen flutamide failed to achieve $50 \%$ inhibition at $\leq 50 \mu \mathrm{M}$, it also appeared to inhibit peptide binding in a concentration-dependent manner (Fig. 6B, D, and Table 2). None of the other test compounds inhibited biotin-TIF2-LXXLL-peptide binding to AR-LBD (Fig. 6B-D and Table 2).

Can Any of the Test Compounds Block AR-NTD (AF-1's) Transcriptional Activity?

We initially set out to develop AF-1 M2H assays by substituting a Gal4-AR-NTD construct for the Gal4-AR-LBD construct used in the M2H assays described above (Supplementary Fig. S6A). However, not only was Gal4-AR-NTDmediated transcriptional activity independent of DHT treatment 


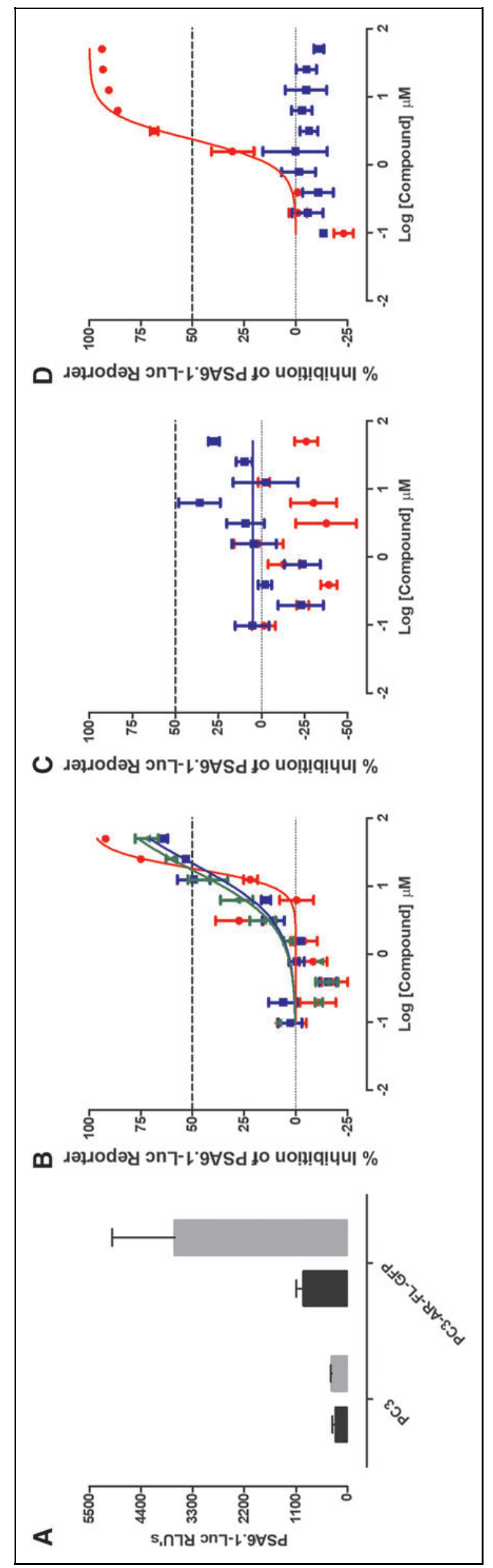

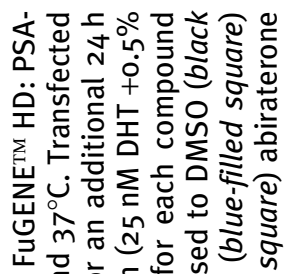

능 든

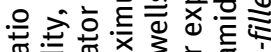

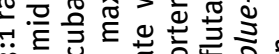

m.

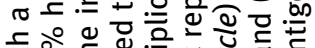

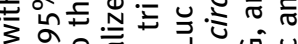

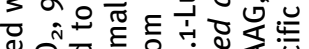

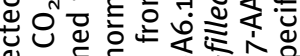

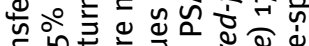

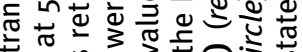

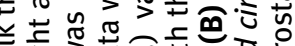

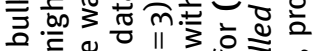

》

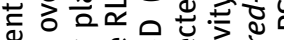

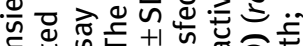

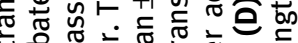

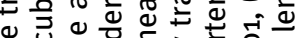

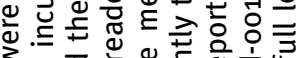

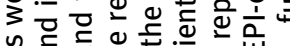

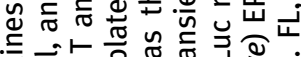

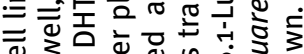

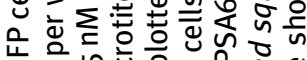

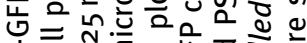

立要

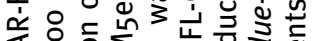

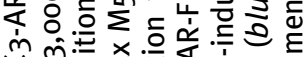

谷

v $\geq 0$

ब

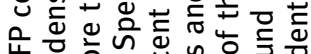

的

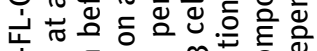

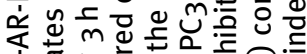

的

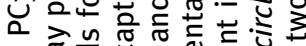

$\subseteq$ 讨

तั

Uू

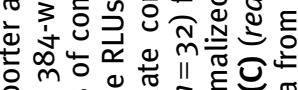

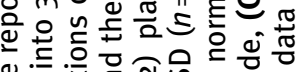

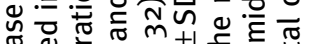

政类

品 $\frac{\pi}{2}$

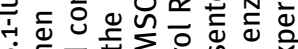

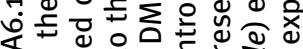

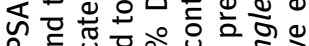

든 웡 웅

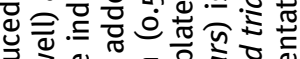

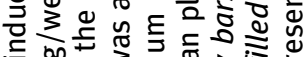

乐 on

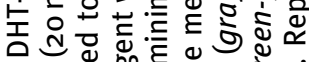

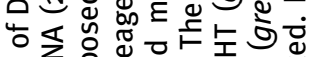

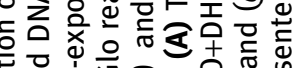

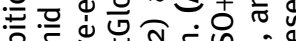

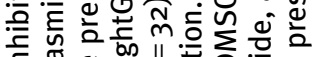

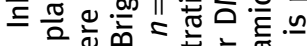

ن

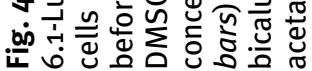




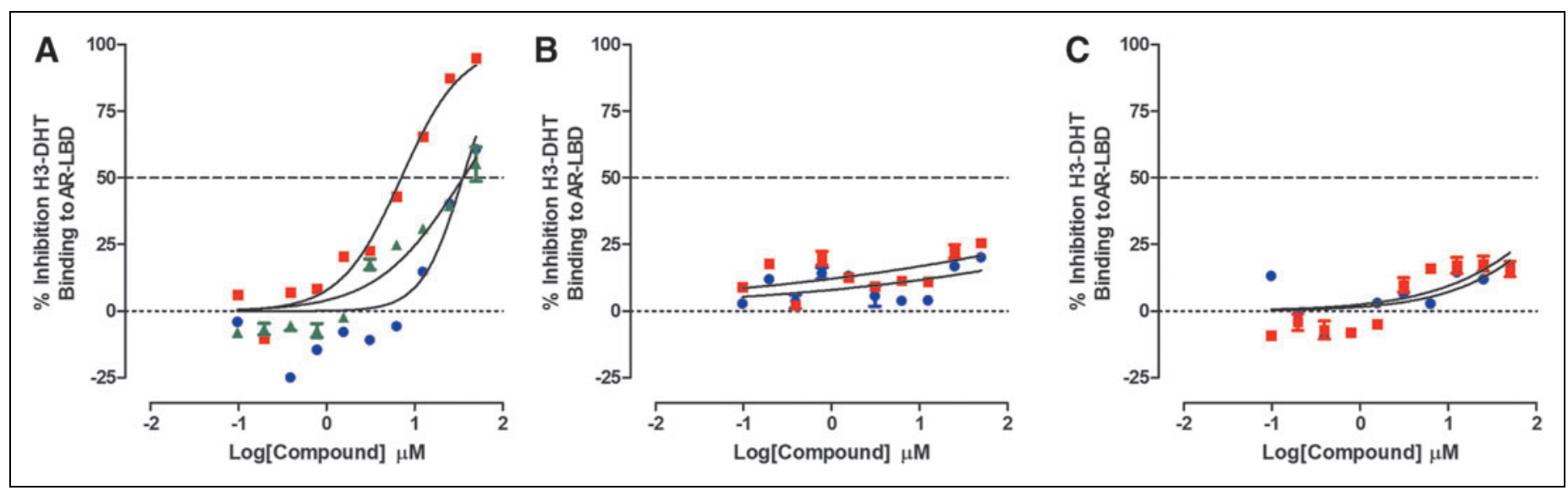

Fig. 5. Inhibition of AR-LBD H3 -DHT Binding. Competitive displacement binding of $\mathrm{H}^{3}$-DHT to His ${ }_{6}$-AR-LBD. Five micrograms of His ${ }_{6}$-AR-LBD protein was added to the wells of 96-well copper-coated plates and allowed to bind overnight at $4^{\circ} \mathrm{C}$. Unbound protein was removed by aspiration; the plate was washed three times with PBS-Tween 20 buffer and blocked for $1 \mathrm{~h} \mathrm{with} 1 \mathrm{mg} / \mathrm{mL}$ BSA in PBS-Tween 20 . After three

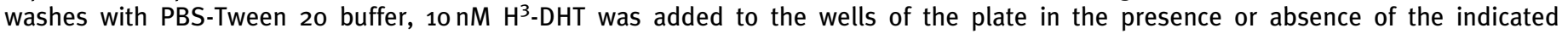
concentrations of test compounds and incubated at RT for $1 \mathrm{~h}$. Unbound $\mathrm{H}^{3}$-DHT was removed by aspiration and washing, and microscintillation reagent was added to the wells, and the CPMs were captured on a TopCount NXT microtiter $\beta$-counter. CPM data were normalized to maximum ( $0.5 \%$ DMSO, $n=8)$ and minimum $(10 \mu \mathrm{M}$ cold DHT, $n=8)$ plate controls, and the percent inhibition was plotted as the mean \pm SD $(n=2)$ values from duplicate wells for each compound concentration. The normalized percent inhibition of the $H^{3}$-DHT bound to $\mathrm{His}_{6}$-AR-LBD for (A) (blue-filled circle) flutamide, (red-filled square) bicalutamide, and (green-filled triangle) enzalutamide, (B) (bluefilled circle) compound No. 10, (red-filled square) EPI-001, (C) (blue-filled circle) 17-AAG, and (red-filled square) abiraterone acetate is presented. Representative experimental data from one of two independent experiments are shown. BSA, bovine serum albumin; CPMs, counts per minute; PBS, phosphate-buffered saline; RT, room temperature.

as expected but it also displayed no apparent requirement for coactivation by either VP16-TIF2 or VP16-SRC1 (Supplementary Fig. S6B). The assay was instead used to interrogate whether compounds could block the constitutive transactivation of $A R-\mathrm{NTD}_{1-503}$. In two independent test compound runs, the pGal4-AR-NTD transactivation assay maximum (DMSO) controls exhibited percent coefficient of variance (\% CVs) $\leq 8.45$ (Supplementary Table S1). All seven test compounds inhibited the constitutive transactivation of the 5xGal4-TATA-Luc reporter by GAL4-AR-NTD ${ }_{1-503}$ (Fig. 7). Surprisingly, flutamide, bicalutamide, and enzalutamide exhibited $\mathrm{IC}_{50}$ of $4.83 \pm 0.14,18.83 \pm 2.46$, and $31.50 \pm 2.63 \mu \mathrm{M}$, respectively, in the NTD transactivation assay, with little or no apparent cytotoxicity (Fig. 7A, D, and Table 3). Similarly, 17-AAG produced an $\mathrm{IC}_{50}$ of $3.54 \pm 0.54 \mu \mathrm{M}$, also with little apparent cytotoxicity (Fig. 7C, F, and Table 3). Although abiraterone acetate produced an $\mathrm{IC}_{50}$ of $6.43 \pm 0.28 \mu \mathrm{M}$ in the NTD transactivation assay, it also was cytotoxic with an $\mathrm{IC}_{50}$ of 30.5 $\pm 7.72 \mu \mathrm{M}$ (Fig. 7C, F, and Table 3). Compound No. 10 and EPI-001 also produced IC $_{50}$ S of $1.55 \pm 0.04 \mu \mathrm{M}$ and $9.92 \pm 1.24 \mu \mathrm{M}$, respectively, in the NTD transactivation assay (Fig. $7 B$ and Table 3), with corresponding $\mathrm{IC}_{50} \mathrm{~S}$ of $26.5 \pm$ $10.9 \mu \mathrm{M}$ and $36.6 \pm 9.3 \mu \mathrm{M}$, respectively, in the associated viability assays, suggesting that compound-mediated cyto- toxicity may be contributing significantly to the decreased reporter signals (Fig. $7 E$ and Table 3).

Do Any of the Test Compounds Inhibit the Constitutive Activity of the PSA-6.1 Promoter Luciferase Reporter Directed by AR-V7?

The PSA6.1 promoter-driven luciferase reporter can be activated by both AR-FL and AR-V7 splice variants that are expressed in PCa cells. ${ }^{24}$ To provide an AF-1-mediated transcriptional activation assay, we transfected the PSA6.1-Luc reporter construct into the AR null PC3 cell line that had been transformed to express AR-V7-EGFP. Supplementary Figure S7A shows representative grayscale DAPI and FITC channel and color composite images of the AR-V7-EGFP-PC3 cell line \pm DHT exposure stained with the Hoechst DNA stain. For comparison, images of the AR null parental PC3 cell line are also presented. Exposure to DHT had no effect on the subcellular localization of the AR-V7-EGFP, which is predominantly nuclear. To compare the PSA6.1 promoter-driven luciferase reporter responses in three distinct cell backgrounds, parental PC3, PC3-AR-FL-EGFP, and PC3-AR-V7EGFP cells were transiently transfected with the PSA6.1-Luc reporter construct, cultured overnight, and then exposed to either DHT (100 nM) or DMSO for $24 \mathrm{~h}$. In parental PC3 cells 

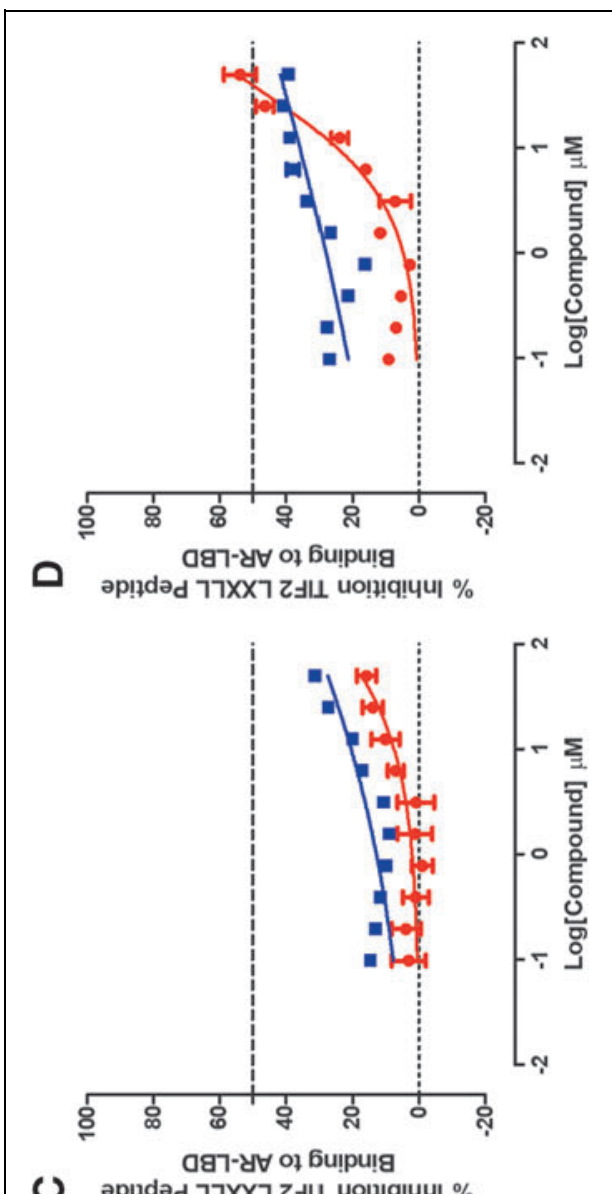

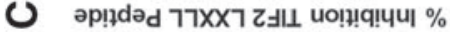

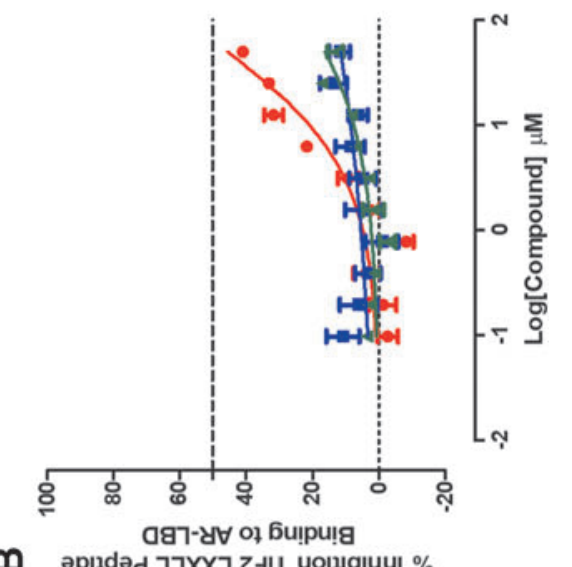

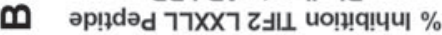
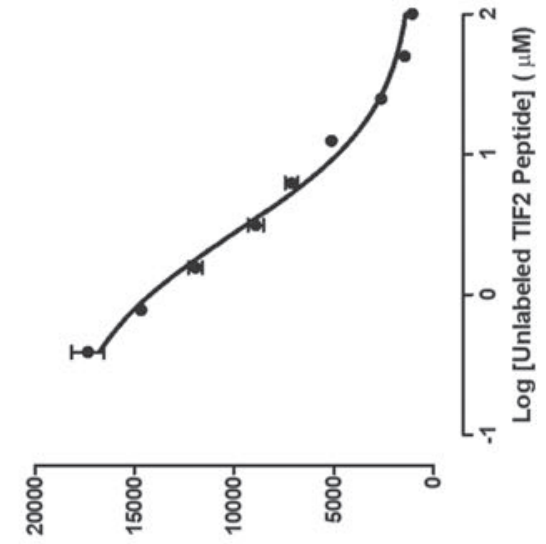

(s, (s)

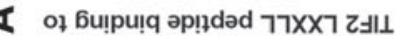

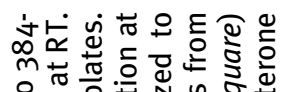

은 는

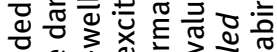

证安过

ब. $\cong \mathrm{m}_{0}^{\infty}$

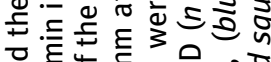

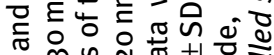

เ゙m

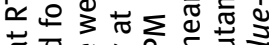

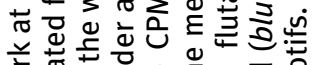

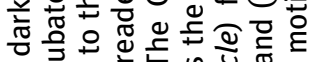

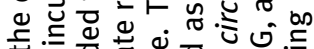

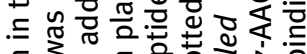

증

E.는

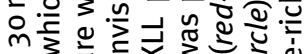

흔

는

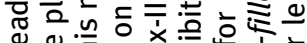

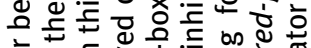

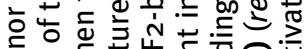

음

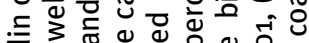

을.

준

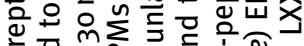

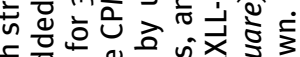

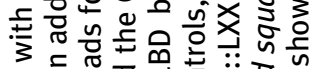

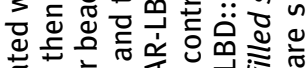

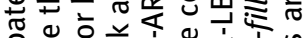

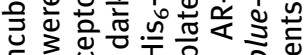
. น

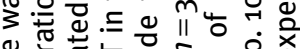

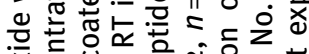

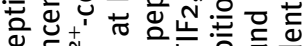

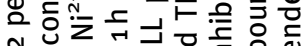

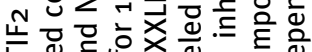

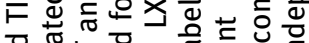

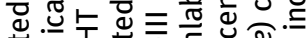

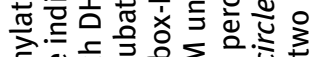

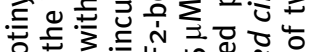

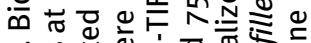
ดो 융 䏠

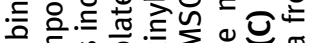

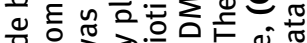
응 政음

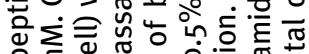
잉

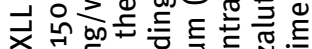

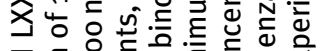

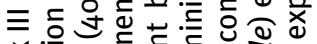

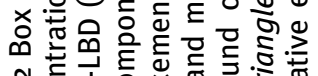
는

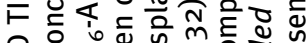
口

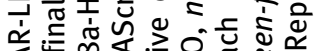

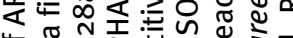

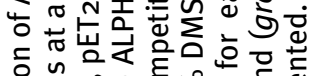
든 을 능

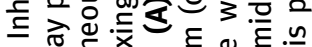

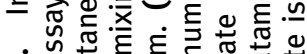
ט。

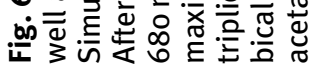



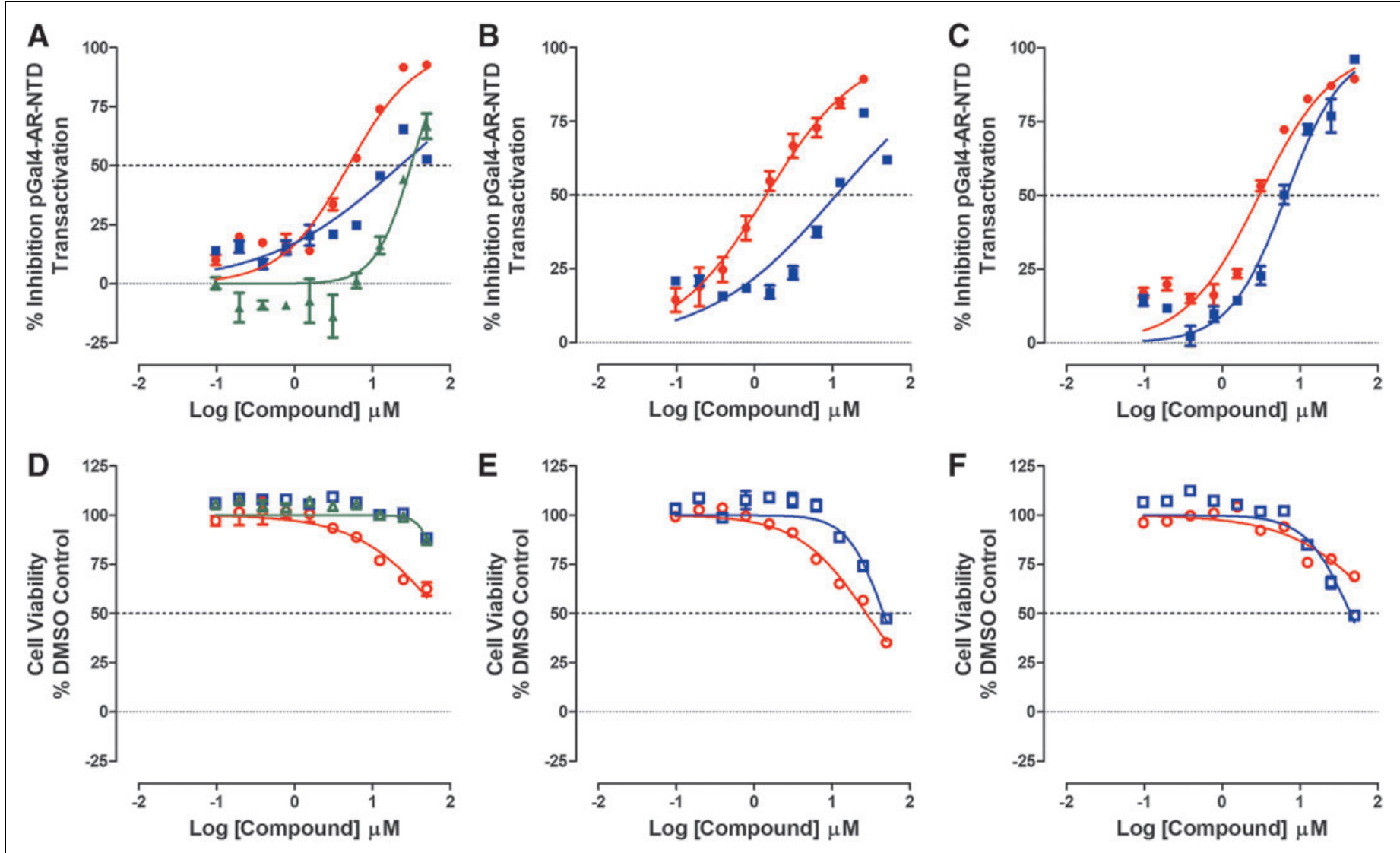

Fig. 7. Inhibition of Constitutive pGal4-AR-NTD Transactivation. HEK 293 cells were cotransfected with two plasmid constructs, pGAL4-ARNTD (1-503) and the $5 \times$ Gal4-TATA-Luc reporter, and 5,000 cells were seeded into the wells of 384-well assay plates, and cultured overnight at $37^{\circ} \mathrm{C}, 5 \% \mathrm{CO}_{2}$, and $95 \%$ humidity. After $24 \mathrm{~h}$, cells were exposed to test compounds at the indicated concentrations and assay plates were returned to the incubator. After an additional $24 \mathrm{~h}$, BrightGlo reagent was added to the plate and the RLUs were captured on a SpectraMax M5e microtiter plate reader. The RLU's data were normalized relative to the maximum ( $0.5 \%$ DMSO, $n=64)$ plate controls, and the percent inhibition was plotted as the mean \pm SD $(n=3)$ values from triplicate wells for each compound concentration. The normalized percent inhibition for treatment with (A) (red-filled circle) flutamide, (blue-filled square) bicalutamide, and (green-filled triangle) enzalutamide, (B) (red-filled circle) compound No. 10, (blue-filled square) EPI-001, (C) (red-filled circle) 17-AAG, and (blue-filled square) abiraterone acetate is presented. The corresponding cell viability data for compound-treated wells were normalized to $0.5 \%$ DMSO control wells $(n=64)$, and the percent cell viability plotted as the mean \pm SD $(n=3)$ values from triplicate wells at each compound concentration for (D) (red open circle) flutamide, (blue open square) bicalutamide, and (green open triangle) enzalutamide, (E) (red open circle) compound No. 10, (blue open square) EPI-001, (F) (red open circle) 17-AAG, and (blue open square) abiraterone acetate is presented. Representative experimental data from one of two independent experiments are shown. AR-NTD, N-terminal domain of AR.

that are null for AR, there was no PSA6.1-Luc reporter response with or without DHT (Supplementary Fig. S7B). In PC3-AR-FL-GFP cells, a strong PSA6.1-Luc reporter response was only observed in cells treated with DHT, whereas in PC3AR-V7-GFP cells, robust constitutive PSA6.1-Luc reporter activity was observed with or without DHT treatment (Supplementary Fig. S7B).

In two independent test compound runs, the PC3-AR-V7EGFP PSA6.1-Luc reporter assay performed well and the maximum (DMSO) controls exhibited \% CVs $\leq 13.1$ (Supplementary Table S1). The AR antagonist flutamide in- hibited AR-V7-driven PSA6.1-Luc reporter activity with an $\mathrm{IC}_{50}$ of $11.5 \pm 1.22 \mu \mathrm{M}$ (Fig. $8 B$ and Table 3). In contrast, neither bicalutamide nor enzalutamide produced an $\mathrm{IC}_{50}$ at $\leq 50 \mu \mathrm{M}$ (Fig. $8 B$ and Table 3). Abiraterone acetate also failed to achieve an $\mathrm{IC}_{50}$ at $\leq 50 \mu \mathrm{M}$ (Fig. $8 D$ and Table 3). 17-AAG produced an $\mathrm{IC}_{50}$ of $0.68 \pm 0.37 \mu \mathrm{M}$ (Fig. $8 D$ and Table 3), and compound No. 10 and EPI-001 exhibited $\mathrm{IC}_{50}$ s of $50.3 \pm 11.3$ and $25.3 \pm 4.05 \mu \mathrm{M}$, respectively (Fig. $8 \mathrm{C}$ and Table 3). None of the test compounds were cytotoxic to PC3-AR-V7-EGFP cells under the conditions used (Table 3). 


\section{ANDROGEN RECEPTOR ACTIVATION FUNCTION DOMAIN ASSAYS}

\begin{tabular}{|c|c|c|c|c|c|c|c|c|c|c|}
\hline \multirow[b]{3}{*}{ Test Compound } & \multicolumn{4}{|c|}{ Transactivation assay $\mathrm{IC}_{50}(\mu \mathrm{M})$} & \multirow{2}{*}{\multicolumn{2}{|c|}{$\begin{array}{c}\text { PSA6.1-Luc } \\
\text { Reporter IC } 50(\mu \mathrm{M}) \\
\text { PC3-AR-V7-GFP }\end{array}$}} & \multirow{2}{*}{\multicolumn{2}{|c|}{$\begin{array}{c}\text { UBE2C-Luc } \\
\text { Reporter IC } \text { C }_{50}(\mu \mathrm{M}) \\
\text { PC3-AR-V7-GFP }\end{array}$}} & \multirow{2}{*}{\multicolumn{2}{|c|}{$\begin{array}{c}\text { Cytotoxicity } \\
\text { PC3-AR-V7-GFP }\end{array}$}} \\
\hline & \multicolumn{2}{|c|}{ pGal4-AR-NTD (1-503) } & \multicolumn{2}{|c|}{ HEK 293 cell Cytox } & & & & & & \\
\hline & Mean & SD & Mean & SD & Mean & SD & Mean & SD & Mean & SD \\
\hline Flutamide & 4.83 & 0.14 & $>50$ & N/A & 11.5 & 1.22 & 28.6 & 2.71 & $>50$ & N/A \\
\hline Bicalutamide & 18.83 & 2.46 & $>50$ & N/A & $>50$ & N/A & $>50$ & $\mathrm{~N} / \mathrm{A}$ & $>50$ & N/A \\
\hline Enzalutamide & 31.5 & 2.63 & $>50$ & N/A & $>50$ & N/A & $>50$ & $\mathrm{~N} / \mathrm{A}$ & $>50$ & N/A \\
\hline Compound-10 & 1.55 & 0.04 & 26.5 & 10.9 & 50.3 & 11.3 & 58.6 & 0.79 & $>50$ & N/A \\
\hline EPI-001 & 9.92 & 1.24 & 36.6 & 9.3 & 25.3 & 4.05 & 49.2 & 0.76 & $>50$ & N/A \\
\hline 17-AAG & 3.54 & 0.54 & $>50$ & N/A & 0.68 & 0.37 & 0.75 & 0.50 & $>50$ & N/A \\
\hline Abiraterone & 6.43 & 0.28 & 30.5 & 7.72 & $>50$ & N/A & $>50$ & $\mathrm{~N} / \mathrm{A}$ & $>50$ & N/A \\
\hline
\end{tabular}

AR-NTD, N-terminal domain of AR; AR-V7, androgen receptor splice variant 7; $p G a \mid 4$, DNA-binding domain of the Gal4 protein derived from yeast; UBE2C, ubiquitinconjugating enzyme E2C.

Do Any of the Test Compounds Inhibit the Constitutive Activity of the UBE2C Promoter Luciferase Reporter Directed by AR-V7?

UBE2C it is a specific target gene of AR splice variants. $^{46,55,59}$ The UBE2C reporter is a luciferase construct driven by three repeats of an AR-V7-specific promoter element from the UBE2C gene. ${ }^{55}$ To provide an AF-1 selective transcriptional activation assay, we transfected the UBE2C-Luc reporter construct into $\mathrm{PC} 3-\mathrm{AR}-\mathrm{V7}$-EGFP cells. To compare the UBE2C promoter-driven luciferase reporter responses in three distinct cell backgrounds, parental PC3, PC3-AR-FLEGFP, and PC3-AR-V7-EGFP cells were transiently transfected with the UB2EC-Luc reporter construct, cultured overnight, and then exposed to either DHT (100 nM) or DMSO for $24 \mathrm{~h}$. In both parental PC3 and PC3-AR-FL-EGFP cells, there was no UBE2C-Luc reporter response either with or without DHT (Supplementary Fig. S7C). In PC3-AR-V7-GFP cells, however, robust constitutive UBE2C-Luc reporter activity was observed with or without DHT treatment (Supplementary Fig. S7C).

In two independent test compound runs, the PC3-AR-V7EGFP UBE2C-Luc reporter assay performed well and the maximum (DMSO) controls exhibited \% CVs $\leq 11.8$ (Fig. 9 and Supplementary Table S1). The antiandrogen flutamide inhibited AR-V7-driven UBE2C-Luc reporter activity with an $\mathrm{IC}_{50}$ value of $28.6 \pm 2.71 \mu \mathrm{M}$, while bicalutamide and enzalutamide failed to achieve an $\mathrm{IC}_{50}$ at $\leq 50 \mu \mathrm{M}$ (Fig. $9 B$ and Table 3). Abiraterone acetate also failed to achieve an $\mathrm{IC}_{50}$ at $\leq 50 \mu \mathrm{M}$ in the AR-V7-driven UBE2C-Luc reporter assay
(Fig. 9D and Table 3). In contrast, the Hsp90 inhibitor 17-AAG produced an $\mathrm{IC}_{50}$ of $0.75 \pm 0.50 \mu \mathrm{M}$ (Fig. 9D and Table 3), and compound No. 10 and EPI-001 exhibited $\mathrm{IC}_{50}$ s of 58.6 \pm 0.79 and $49.2 \pm 0.76 \mu \mathrm{M}$, respectively, in the AR-V7-driven UBE2C-Luc reporter assay (Fig. 9C and Table 3). However, compound No. 10 and EPI-001 were only active at the highest concentration used in the assay. None of the test compounds were cytotoxic to PC3-AR-V7-EGFP cells under the conditions used (Table 3).

\section{DISCUSSION}

Increased expression of $\mathrm{AR}$ coactivators and $\mathrm{AR}$ splice variants occurs in PCa patients who have relapsed on ADT and contributes to poor CRPC patient outcomes by increasing $A R$ transcriptional activity under androgen-depleted conditions. ${ }^{13,16,19-21}$ The AF-2 and AF-1 transactivation domains serve as binding surfaces for coactivator proteins that facilitate AR gene transcription. ${ }^{14,15,22-25,33-36}$ Coactivator interactions with both AR-FL and splice variants compensate for low cellular DHT levels after ADT treatment and result in persistent transcriptional activity. ${ }^{13-16}$ The AF-2 and AF-1 binding surfaces mediate androgen-dependent and androgenindependent transactivation, respectively. AR-V7 splice variants, which are upregulated in CRPC patients who have relapsed on ADT, only contain an AF-1 surface. ${ }^{14,15,34,40,42}$ The ideal inhibitor/disruptor of AR-coactivator PPIs for CRPC therapy would target both the AF-2 and AF-1 surfaces and effectively block transcriptional activation by both AR-FL and splice variants. We recently implemented an AR-TIF2 PPIB 


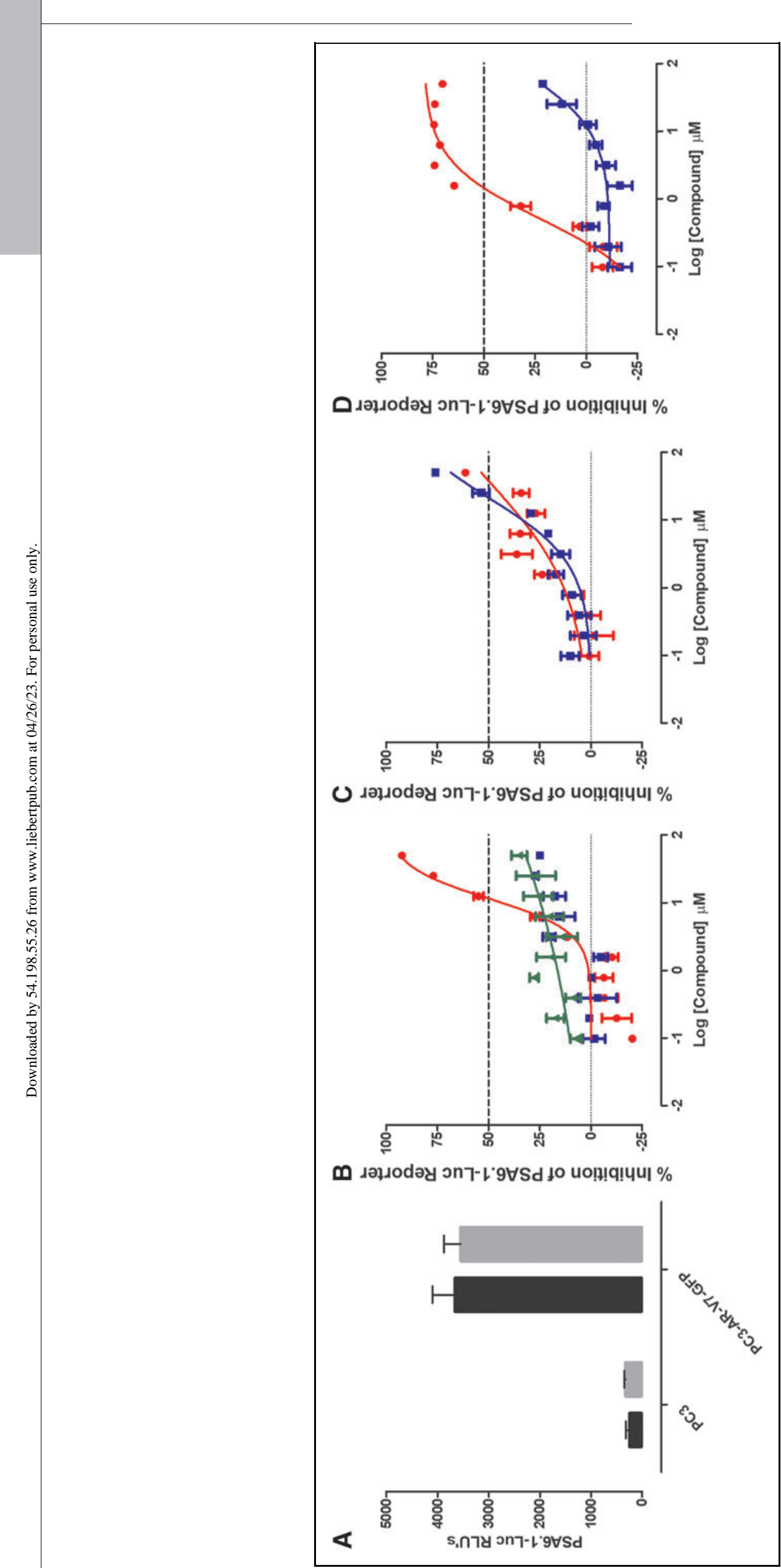

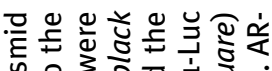

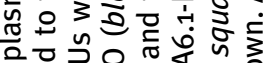
웅

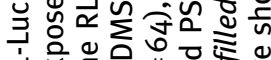
붕 ख

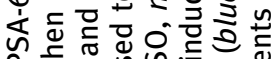
药

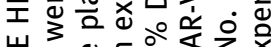

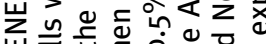
嵌

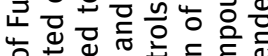

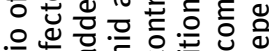
일

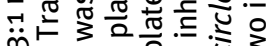

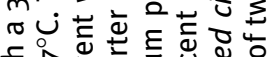

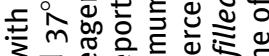

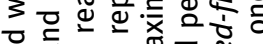

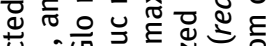

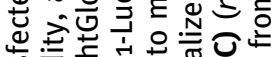
ज. 항 흔 도 인 중 $\frac{\pi}{0}$

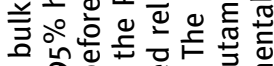
> 产 $0^{\hat{N}}$

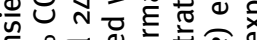

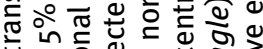

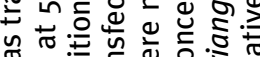

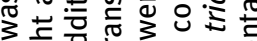
o 至

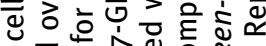

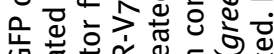
둥

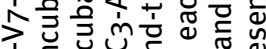
这.气

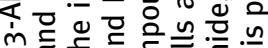
政宁

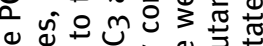

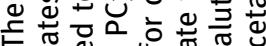
응

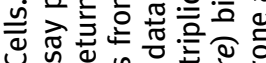
น 눙 今3 3

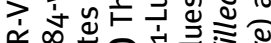

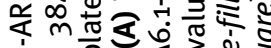
ن். 뭉 $\subseteq 3$ 㟧

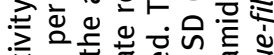

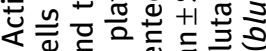

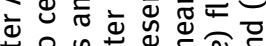

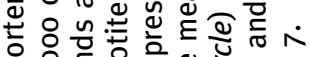
웡

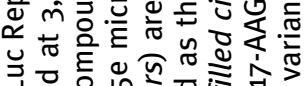

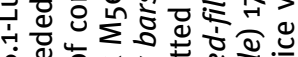
过范 ऊ

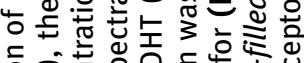

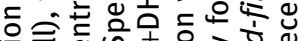

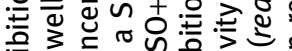
空 on

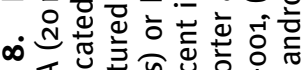

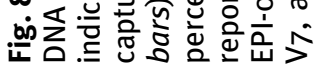



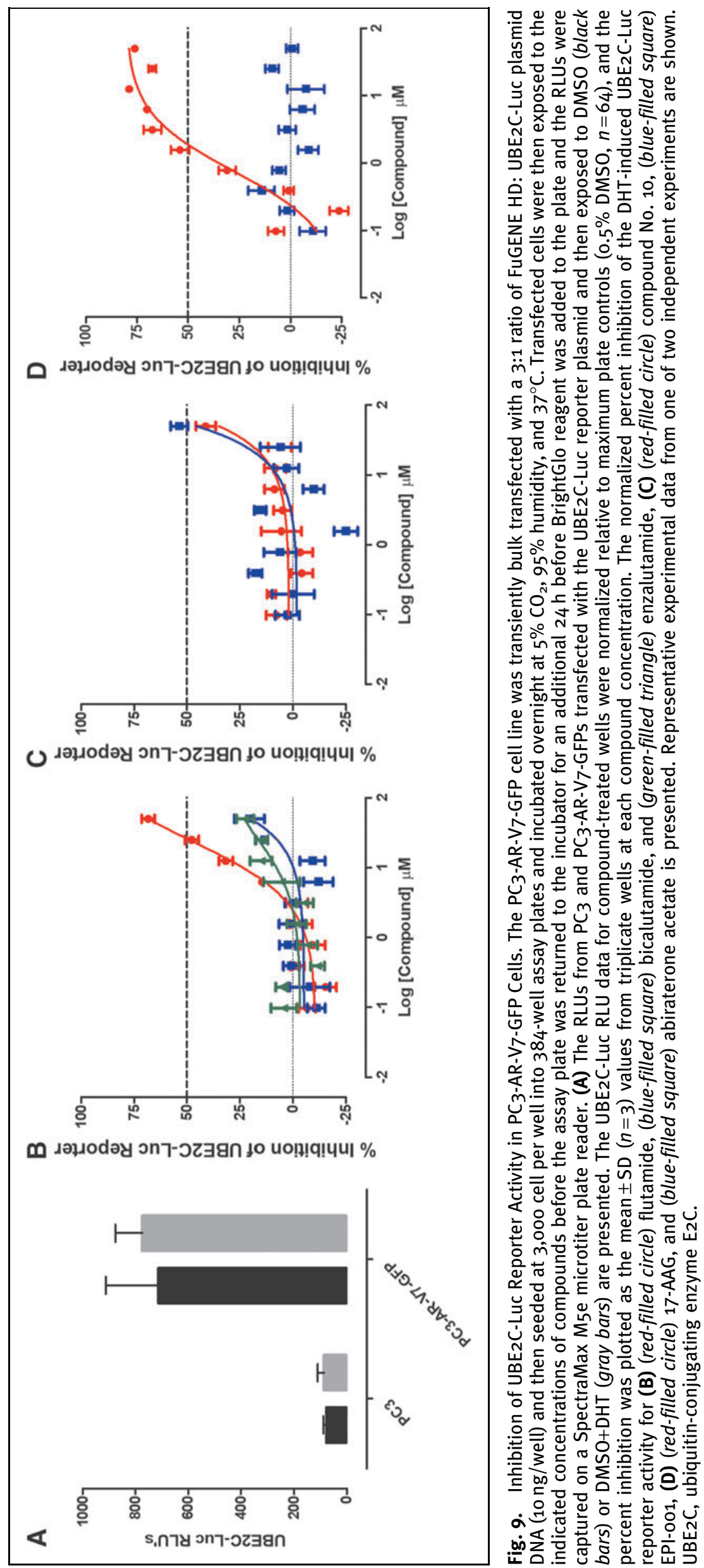
HCS campaign of $\geq 143,000$ compounds to identify hits that inhibit or disrupt the interactions between AR and the TIF2 coactivator. ${ }^{2-25}$ Since the AR-TIF2 PPIB design incorporates only the AR-LBD region of AR and the region of the TIF2 coactivator's NID that contains the box III LXXLL motif, we were concerned that hits might be biased toward the AF-2 surface. We therefore developed a panel of AF-2- and AF-1focused assays to characterize the transactivation domain profiles of our hits and to prioritize those that were effective at blocking coactivator recruitment at both surfaces (Supplementary Table S2).

To validate our AF-2 and AF-1 assays, we assembled a test set of four FDA-approved ADTs, two investigational molecules that target the AR-NTD, and an inhibitor of the Hsp90 molecular chaperone. Flutamide, bicalutamide, and enzalutamide are AR-antagonists that are prescribed as components of an ADT strategy (Supplementary Fig. S2). ${ }^{60-62}$ Similarly, the Cyp 17a1 inhibitor abiraterone acetate, which blocks androgen synthesis, is also used as a component of ADT. ${ }^{63}$ Compounds No. 10 and EPI-001 target the amino terminus of AR rather than the ligand binding site, ${ }^{30,41,48,49,64}$ and 17-AAG is an inhibitor of the Hsp90 molecular chaperone ATPase that is involved in the folding, maturation, and maintenance of AR in a high-affinity ligand binding conformation (Supplementary Fig. S2) ${ }^{65}$ Consistent with previous studies, ${ }^{22-25}$ all three AR antagonists and the Hsp90 inhibitor 17-AAG effectively inhibited DHT-induced AR-TIF2 PPI formation and disrupted preformed AR-TIF2 complexes in a concentration-dependent manner (Fig. 1 and Table 1). In contrast, compound No. 10, EPI-001, or abiraterone acetate did not produce a calculable $\mathrm{IC}_{50}$ in either format of the AR-TIF2 PPIB assay (Fig. 1 and Table 1). Since compound No. 10 and EPI-001 target the ARNTD, which is not part of the AR-RFP biosensor, and abiraterone acetate inhibits Cyp17a1, an enzyme involved with androgen biosynthesis, it was expected that these compounds would not be active in the AR-TIF2 biosensor. Although compound No. 10 failed to produce a calculable $\mathrm{IC}_{50}$, it inhibited DHT-induced AR-TIF2 PPI formation, potentially due to its ability to reduce AR expression levels and/or restrict its localization to the cytoplasm..$^{30,48,49}$ None of the test compounds were acutely cytotoxic toward U-2 OS cells under the conditions used.

We then profiled the activities of the test compounds in five AF-2-focused assays (Figs. 2-6 and Table 2). M2H assays represent the gold standard method for measuring how NR interacts with coregulator proteins to modulate NR transcriptional activity. ${ }^{66-69}$ SRC1 and TIF2 are members of the steroid NR p160 coactivator family, ${ }^{70,71}$ and similar to TIF2, increased expression of SRC1 has been associated with recurrent PCa. ${ }^{13,70-73}$ Based on the activity profiles of the test compounds in the AR-TIF2 PPIB assay, we anticipated that the three AR antagonists and 17-AAG would also be active in the two M2H assays. It was surprising therefore that all seven test compounds inhibited DHT-induced AR-LBD interactions with the TIF2 and SRC1 coactivators in a concentrationdependent manner (Figs. 1-3 and Table 2). However, abiraterone acetate, compound No. 10, and EPI-001 also displayed substantial concentration-dependent cytotoxicity in HEK 293 cells, which may have contributed to their apparent activity in the $\mathrm{M} 2 \mathrm{H}$ assays. None of the test compounds were selective in their ability to disrupt DHT-induced AR-LBD M2H interactions with either TIF2 or SRC1. We anticipated that AR-TIF2 PPI inhibitors/disruptors would effectively block DHT-induced transactivation, and indeed, only the three AR antagonists and 17-AAG inhibited DHTinduced AR-FL-GFP PSA6.1-luciferase reporter activity in a concentration-dependent manner (Fig. 4 and Table 2). As expected, the anti-Cyp17a1 inhibitor abiraterone acetate did not inhibit DHT-induced AR-FL-GFP PSA6.1-luciferase reporter activity. The two AR-NTD-targeted compounds, compound No. 10 and EPI-001, also did not inhibit ligandactivated AR-FL transcription. Reassuringly, the three antiandrogen AR antagonists flutamide, bicalutamide, and enzalutamide competitively displaced $\mathrm{H}^{3}$-DHT binding to AR-LBD, and produced calculable $\mathrm{IC}_{50} \mathrm{~S}$ in all the AF-2 surface assays that utilized DHT to induce a response (Fig. 5 and Table 2). As anticipated, the Hsp90 inhibitor 17-AAG, the Cyp17a1 inhibitor abiraterone acetate, and the two AR-NTDtargeted compounds did not inhibit $\mathrm{H}^{3}$-DHT binding to the AR-LBD, nor were they active in any of the AF-2-focused assays, except for the M2H assays as discussed above (Fig. 5 and Table 2). Our original intent had been to use the AR-LBD $\mathrm{H}^{3}$-DHT binding assay to identify and deprioritize AR-TIF2 PPI inhibitor/disruptor hits that were AR antagonists, in part, not only because of the number of existing FDA-approved PCa drugs with this mechanism of action but also because the inevitable development of drug resistance to such drugs limits the duration of their efficacy for CRPC. ${ }^{5,11,13}$ However, it is possible that a compound, which binds to an allosteric binding site to induce a conformational change, that blocks AR-TIF2 PPIs might also have a negative impact on orthosteric ligand binding. ${ }^{74}$ Indeed, an allosteric modulator that blocked AR-TIF2 PPIs and ligand binding would have a desirable bioactivity profile. The AF-2 surface interacts with coactivators containing binding motifs encoded by LXXLL and/or FXXLF sequences to regulate androgen-dependent AR transactivation. ${ }^{14,37-39}$ Based on their established targets 
and mechanisms of action, we did not anticipate that any of the test compounds would block TIF2 Box III LXXLL peptide binding to the AR-LBD (Fig. 6 and Table 2). However, the Hsp90 inhibitor 17-AAG produced a calculable $\mathrm{IC}_{50}$ and flutamide displayed evidence of concentration-dependent inhibition of TIF2-LXXLL-peptide binding to the AR-LBD. We used the SwissADME program hosted by the Swiss Institute of Bioinformatics to evaluate whether any of our test compounds might be pan-assay interference compounds (PAINS) ${ }^{75}$ that interfere with the ALPHAScreen assay format. Only 17-AAG was flagged as a potential PAINS due to the presence of a quinone within the structure. Interestingly, only the Hsp90 inhibitor 17-AAG displayed the desired bioactivity profile of an AR-TIF2 PPI inhibitor/disruptor; it inhibited the AR-TIF2 PPIB assay and produced calculable $\mathrm{IC}_{50} \mathrm{~S}$ in all the AF-2 surface assays, but not an AR antagonist. However, since 17-AAG is an Hsp90 ATPase inhibitor, its activity in the AR-TIF2 PPIB and AF-2-focused assays was likely due to its ability to block the Hsp90-mediated maturation, by folding and maintenance of AR in a high-affinity conformation for ligand binding, and/or by blocking AR nuclear trafficking.

The AF-1-focused assays included a ligand-independent AR-NTD transactivation assay conducted in HEK 293 cells, and the PSA6.1 and UBE2C promoter-driven luciferase reporter assays conducted in PC3-AR-V7-GFP cells. Unexpectedly, we observed that the pGal4-AR-NTD construct did not respond to either SRC1 or TIF2 coactivation (Supplementary Fig. S6B), which was inconsistent with the published reports. ${ }^{14,15,34,42,76,77}$ There were, however, some differences in how our assays were configured compared to those other studies. The other groups conducted their assays in CV-1 cells, and the $\mathrm{M} 2 \mathrm{H}$ bait and prey constructs were flipped with the TIF2 and SRC1 coactivators being inserted into the Gal4-DBD plasmids and AR-NTD inserted into the VP16 vector. We, therefore, used our assay to measure constitutive AR-NTDmediated transactivation. Surprisingly, all seven test compounds inhibited constitutive AR-NTD transactivation in a concentration-dependent manner (Fig. 7 and Table 3). Once again, however, abiraterone acetate, compound No. 10, and EPI-001 also displayed substantial concentration-dependent cytotoxicity in HEK 293 cells, which may have contributed to their apparent activity in the AR-NTD transactivation assay. In the two reporter assays conducted in PC3-AR-V7-GFP cells, only four compounds produced calculable $\mathrm{IC}_{50} \mathrm{~s}$ : compound No. 10, EPI-001, 17-AAG, and flutamide (Figs. 8 and 9, and Table 3). It was reassuring that compound No. 10 and EPI-001, which target the AR-NTD, inhibited AR-V7-induced reporter activity. It is possible that the Hsp90 molecular chaperone inhibitor 17-AAG was active in both reporter assays because of its ability to inhibit the maturation, folding, and activity of the luciferase enzyme; however, the ability of the AR antagonist flutamide to inhibit AR-V7-driven reporter responses was unexpected.

PCa progression to CRPC requires continued AR signaling even after treatment with ADTs. Overexpression of AR coactivators and the emergence of AR splice variants both promote continued transcriptional activation under androgen-depleted conditions, which constitute drug resistance mechanisms contributing to CRPC progression. We have prosecuted an HCS campaign to identify hit compounds that can inhibit or disrupt the PPIs between AR and TIF2, one of the coactivators implicated in CRPC disease progression. ${ }^{22-}$ ${ }^{25}$ Since an ideal inhibitor/disruptor of AR coactivator PPIs would target both the AF-2 and AF-1 domains, which serve as binding surfaces for coactivators, we have developed and validated a panel of assays to interrogate and prioritize hits that disrupt both transactivation surfaces (article in preparation). Five $\mathrm{AF}-2$ - and three $\mathrm{AF}-1$-focused assays were developed. The TIF2 and SRC1 M2H assays will be used to confirm that AR-TIF2 PPIB hits block AR AF-2 coactivator interactions and to evaluate p160 coactivator selectivity. The PSA6.1-Luc reporter assay conducted in PC3-AR-GFP cells will confirm that hits block ligand-dependent AR-FL transcriptional activation. The AR-LBD $\mathrm{H}^{3}$-DHT binding assay will enable us to identify hits that are AR antagonists, or that may be negative allosteric modulators that also affect ligand binding. The AR-LBD TIF2 Box III LXXLL peptide binding assay will enable us to identify hits that inhibit/ disrupt an established mechanism of coactivator binding to the AF-2 surface. The constitutive AR-NTD transactivation assay will allow us to test whether hits can also inhibit ligand-independent AF-1-mediated transcriptional activation, and the PSA6.1 and UBE2C reporter assays conducted in PC3-AR-V7-GFP cells will be used to determine if hits can also block ligand-independent splice variant transcriptional activation. AR-TIF2 PPI inhibitor/disruptor hits that can inhibit coactivator recruitment and AR transcriptional activation at both the AF- 2 and AF- 1 surfaces would be prioritized for lead optimization and have the potential to be developed into novel drug candidates for CRPC therapy.

\section{ACKNOWLEDGMENTS}

We would like to thank Dr. Zhou Wang from the Department of Urology of the University of Pittsburgh Hillman Cancer Institute for providing us with reagents and compound 10 required to conduct our studies. We would also like to 
thank Daniel Camarco and David Close from the Department of Pharmaceutical Sciences of the University of Pittsburgh for their expert technical assistance.

\section{DISCLOSURE STATEMENT}

The authors declare no conflicts of interest.

\section{FUNDING INFORMATION}

The studies reported herein were funded by grant support from the National Institutes of Health (NIH); R21NS073889 Johnston (principal investigator [PI]) from the National Institute of Neurological Disorders and Stroke (NINDS), and R01CA160423 and R01CA183882 Johnston (PI) from the National Cancer Institute (NCI).

\section{SUPPLEMENTARY MATERIAL}

\author{
Supplementary Figure S1 \\ Supplementary Figure S2 \\ Supplementary Figure S3 \\ Supplementary Figure S4 \\ Supplementary Figure S5 \\ Supplementary Figure S6 \\ Supplementary Figure S7 \\ Supplementary Table S1 \\ Supplementary Table S2
}

\section{REFERENCES}

1. Siegel RL, Miller KD, Jemal A: Cancer statistics, 2018. CA Cancer J Clin 2018;68: 7-30.

2. Kirby $M$, Hirst $C$, Crawford ED: Characterising the castration-resistant prostate cancer population: a systematic review. Int J Clin Pract 2011;65:1180-1192.

3. Keyes M, Crook J, Morton G, Vigneault E, Usmani N, Morris WJ: Treatment options for localized prostate cancer. Can Fam Physician 2013;59:1269-1274.

4. Gudziak MR, Smith AY: Hormonal therapy for stage $D$ cancer of the prostate. West J Med 1994;160:351-359.

5. Harris WP, Mostaghel EA, Nelson PS, Montgomery B: Androgen deprivation therapy: progress in understanding mechanisms of resistance and optimizing androgen depletion. Nat Clin Pract Urol 2009;6:76-85.

6. Thompson IM: Flare associated with LHRH-agonist therapy. Rev Urol 2001; 3(Suppl 3):S10-S14.

7. Agoulnik IU, Weigel NL: Androgen receptor action in hormone-dependent and recurrent prostate cancer. J Cell Biochem 2006;99:362-372.

8. Scher HI, Fizazi $K$, Saad $F$, et al.: Increased survival with enzalutamide in prostate cancer after chemotherapy. N Engl J Med 2012;367:1187-1197.

9. de Bono JS, Logothetis CJ, Molina A, et al.: Abiraterone and increased survival in metastatic prostate cancer. N Engl J Med 2011;364:1995-2005.

10. Liu LL, Xie N, Sun S, Plymate $S$, Mostaghel E, Dong X: Mechanisms of the androgen receptor splicing in prostate cancer cells. Oncogene 2014;33:31403150.

11. Karantanos T, Corn PG, Thompson TC: Prostate cancer progression after androgen deprivation therapy: mechanisms of castrate resistance and novel therapeutic approaches. Oncogene 2013;32:5501-5511.

12. Waltering KK, Urbanucci $A$, Visakorpi T: Androgen receptor (AR) aberrations in castration-resistant prostate cancer. Mol Cell Endocrinol 2012;360:38-43.
13. Gregory $\mathrm{CW}, \mathrm{He} B$, Johnson $\mathrm{RT}$, et al.: A mechanism for androgen receptormediated prostate cancer recurrence after androgen deprivation therapy. Cancer Res 2001;61:4315-4319.

14. Bevan CL, Hoare S, Claessens F, Heery DM, Parker MG: The AF1 and AF2 domains of the androgen receptor interact with distinct regions of SRC1. Mol Cell Biol 1999;19:8383-8392.

15. Christiaens V, Bevan CL, Callewaert $L$, et al.: Characterization of the two coactivator-interacting surfaces of the androgen receptor and their relative role in transcriptional control. J Biol Chem 2002;277:49230-49237.

16. Shi $X B$, Xue L, Zou JX, Gandour-Edwards $R$, Chen $H$, deVere White RW: Prolonged androgen receptor loading onto chromatin and the efficient recruitment of p160 coactivators contribute to androgen-independent growth of prostate cancer cells. Prostate 2008;68:1816-1826.

17. Grossmann ME, Huang $H$, Tindall DJ: Androgen receptor signaling in androgenrefractory prostate cancer. J Natl Cancer Inst 2001;93:1687-1697.

18. Dehm SM, Tindall DJ: Molecular regulation of androgen action in prostate cancer. J Cell Biochem 2006:99:333-344.

19. Agoulnik IU, Vaid $A$, Nakka $M$, et al.: Androgens modulate expression of transcription intermediary factor 2 , an androgen receptor coactivator whose expression level correlates with early biochemical recurrence in prostate cancer. Cancer Res 2006;66:10594-10602.

20. Culig Z, Santer FR: Androgen receptor co-activators in the regulation of cellular events in prostate cancer. World J Urol 2012;30:297-302.

21. Fujimoto $\mathrm{N}$, Miyamoto $\mathrm{H}$, Mizokami $\mathrm{A}$, et al.: Prostate cancer cells increase androgen sensitivity by increase in nuclear androgen receptor and androgen receptor coactivators; a possible mechanism of hormone-resistance of prostate cancer cells. Cancer Invest 2007;25:32-37.

22. Hua Y, Camarco DP, Strock CJ, Johnston PA: High content positional biosensor assay to screen for compounds that prevent or disrupt androgen receptor and transcription intermediary factor 2 protein-protein interactions. Methods $\mathrm{Mol}$ Biol 2018;1683:211-227.

23. Hua $Y$, Shun $T Y$, Strock $C$, Johnston PA: High-content positional biosensor screening assay for compounds to prevent or disrupt androgen receptor and transcriptional intermediary factor 2 protein-protein interactions. Assay Drug Dev Technol 2014;12:395-418.

24. Fancher AT, Hua $Y$, Camarco DP, Close DA, Strock CJ, Johnston PA: Reconfiguring the AR-TIF2 protein-protein interaction HCS assay in prostate cancer cells and characterizing the hits from a LOPAC screen. Assay Drug Dev Technol 2016;14:453-477.

25. Fancher AT, Hua Y, Camarco DP, Close DA, Strock CJ, Johnston PA: Highcontent screening campaign to identify compounds that inhibit or disrupt androgen receptor-transcriptional intermediary factor 2 protein-protein interactions for the treatment of prostate cancer. Assay Drug Dev Technol 2018;16:297-319.

26. Daghestani HN, Zhu G, Johnston PA, Shinde SN, Brodsky JL, Day BW: Characterization of inhibitors of glucocorticoid receptor nuclear translocation: a model of cytoplasmic dynein-mediated cargo transport. Assay Drug Dev Technol 2012;10:46-60.

27. Dudgeon DD, Shinde SN, Shun TY, et al.: Characterization and optimization of a novel protein-protein interaction biosensor high-content screening assay to identify disruptors of the interactions between $\mathrm{p} 53$ and hDM2. Assay Drug Dev Technol 2010;8:437-458.

28. Dudgeon DD, Shinde $S$, Hua Y, et al.: Implementation of a 220,000-compound HCS campaign to identify disruptors of the interaction between p53 and hDM2 and characterization of the confirmed hits. J Biomol Screen 2010;15: 766-782.

29. Hua $Y$, Strock $C J$, Johnston PA: High content screening biosensor assay to identify disruptors of p53-hDM2 protein-protein interactions. Methods Mol Biol 2015:1278:555-565.

30. Johnston PA, Nguyen MM, Dar JA, et al.: Development and implementation of a high-throughput high-content screening assay to identify inhibitors of androgen receptor nuclear localization in castration-resistant prostate cancer cells. Assay Drug Dev Technol 2016;14:226-239. 
31. Johnston PA, Shinde SN, Hua Y, Shun TY, Lazo JS, Day BW: Development and validation of a high-content screening assay to identify inhibitors of cytoplasmic dynein-mediated transport of glucocorticoid receptor to the nucleus. Assay Drug Dev Technol 2012;10:432-456.

32. Htun H, Barsony J, Renyi I, Gould DL, Hager GL: Visualization of glucocorticoid receptor translocation and intranuclear organization in living cells with a green fluorescent protein chimera. Proc Natl Acad Sci U S A 1996;93:48454850.

33. Berrevoets CA, Doesburg P, Steketee K, Trapman J, Brinkmann A0: Functional interactions of the AF-2 activation domain core region of the human androgen receptor with the amino-terminal domain and with the transcriptional coactivator TIF2 (transcriptional intermediary factor2). Mol Endocrinol 1998; 12:1172-1183.

34. Callewaert L, Van Tilborgh N, Claessens F: Interplay between two hormoneindependent activation domains in the androgen receptor. Cancer Res 2006;66: 543-553.

35. Hur E, Pfaff SJ, Payne ES, Gron H, Buehrer BM, Fletterick RJ: Recognition and accommodation at the androgen receptor coactivator binding interface. PLOS Biol 2004;2:E274

36. Moras $D$, Gronemeyer $H$ : The nuclear receptor ligand-binding domain: structure and function. Curr Opin Cell Biol 1998;10:384-391.

37. Dubbink HJ, Hersmus $R$, Verma CS, et al.: Distinct recognition modes of FXXLF and LXXLL motifs by the androgen receptor. Mol Endocrinol 2004;18:21322150.

38. He $B$, Minges JT, Lee LW, Wilson EM: The FXXLF motif mediates androgen receptor-specific interactions with coregulators. J Biol Chem 2002;277:1022610235.

39. Dubbink $H J$, Hersmus $R$, Pike $A C$, et al.: Androgen receptor ligand-binding domain interaction and nuclear receptor specificity of FXXLF and LXXLL motifs as determined by L/F swapping. Mol Endocrinol 2006;20:1742-1755.

40. Lavery DN, McEwan IJ: Structure and function of steroid receptor AF1 transactivation domains: induction of active conformations. Biochem J 2005; 391(Pt 3):449-464.

41. De Mol E, Fenwick RB, Phang CT, et al.: EPI-001, a compound active against castration-resistant prostate cancer, targets transactivation unit 5 of the androgen receptor. ACS Chem Biol 2016;11:2499-2505.

42. Ueda T, Mawji NR, Bruchovsky N, Sadar MD: Ligand-independent activation of the androgen receptor by interleukin- 6 and the role of steroid receptor coactivator-1 in prostate cancer cells. J Biol Chem 2002;277:38087-38094.

43. Wilson EM: Analysis of interdomain interactions of the androgen receptor Methods Mol Biol 2011;776:113-129.

44. Sharp A, Welti J, Blagg J, de Bono JS: Targeting androgen receptor aberrations in castration-resistant prostate cancer. Clin Cancer Res 2016;22:4280-4282.

45. Krause WC, Shafi AA, Nakka M, Weigel NL: Androgen receptor and its splice variant, $A R-V 7$, differentially regulate FOXA1 sensitive genes in $\mathrm{LNCaP}$ prostate cancer cells. Int J Biochem Cell Biol 2014;54:49-59.

46. Hu R, Lu C, Mostaghel EA, et al.: Distinct transcriptional programs mediated by the ligand-dependent full-length androgen receptor and its splice variants in castration-resistant prostate cancer. Cancer Res 2012;72:3457-3462.

47. Johnson JK, Skoda EM, Zhou J, et al.: Small molecule antagonists of the nuclear androgen receptor for the treatment of castration-resistant prostate cancer. ACS Med Chem Lett 2016;7:785-790.

48. Masoodi $K Z$, Eisermann $K$, Yang $Z$, et al.: Inhibition of androgen receptor function and level in castration-resistant prostate cancer cells by 2-[(isoxazol4-ylmethyl)thio]-1-(4-phenylpiperazin-1-yl)ethanone. Endocrinology 2017;158: 3152-3161.

49. Masoodi $K Z, X u Y, D a r ~ J A$, et al.: Inhibition of androgen receptor nuclear localization and castration-resistant prostate tumor growth by pyrroloimidazole-based small molecules. Mol Cancer Ther 2017;16:21202129 .

50. Gonzalez GA, Montminy MR: Cyclic AMP stimulates somatostatin gene transcription by phosphorylation of CREB at serine 133. Cell 1989;59:675-680.
51. Askew EB, Gampe RT, Jr., Stanley TB, Faggart JL, Wilson EM: Modulation of androgen receptor activation function 2 by testosterone and dihydrotestosterone. J Biol Chem 2007;282:25801-25816.

52. He B, Kemppainen JA, Voegel JJ, Gronemeyer H, Wilson EM: Activation function 2 in the human androgen receptor ligand binding domain mediates interdomain communication with the NH(2)-terminal domain. J Biol Chem 1999;274:3721937225.

53. Eisermann K, Dar JA, Dong J, Wang D, Masoodi KZ, Wang Z: Poly (A) binding protein cytoplasmic 1 is a novel co-regulator of the androgen receptor. PLOS One 2015;10:e0128495.

54. Feau C, Arnold LA, Kosinski A, Guy RK: A high-throughput ligand competition binding assay for the androgen receptor and other nuclear receptors. J Biomol Screen 2009;14:43-48.

55. Xu D, Zhan Y, Qi Y, et al.: Androgen receptor splice variants dimerize to transactivate target genes. Cancer Res 2015;75:3663-3671.

56. Zhou XE, Suino-Powell KM, Li J, et al.: Identification of SRC3/AIB1 as a preferred coactivator for hormone-activated androgen receptor. J Biol Chem 2010;285: 9161-9171.

57. Estebanez-Perpina E, Moore JM, Mar E, et al.: The molecular mechanisms of coactivator utilization in ligand-dependent transactivation by the androgen receptor. J Biol Chem 2005;280:8060-8068.

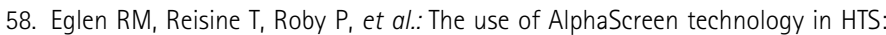
current status. Curr Chem Genomics 2008;1:2-10.

59. Cao B, Qi Y, Zhang G, et al.: Androgen receptor splice variants activating the full-length receptor in mediating resistance to androgen-directed therapy. Oncotarget 2014;5:1646-1656.

60. Goldspiel BR, Kohler DR: Flutamide: an antiandrogen for advanced prostate cancer. DICP 1990;24:616-623.

61. Goa KL, Spencer CM: Bicalutamide in advanced prostate cancer. A review. Drugs Aging 1998;12:401-422.

62. Beer TM, Tombal B: Enzalutamide in metastatic prostate cancer before chemotherapy. N Engl J Med 2014;371:1755-1756.

63. Rehman Y, Rosenberg JE: Abiraterone acetate: oral androgen biosynthesis inhibitor for treatment of castration-resistant prostate cancer. Drug Des Devel Ther 2012;6:13-18.

64. Yang YC, Banuelos CA, Mawji NR, et al.: Targeting androgen receptor activation function-1 with EPI to overcome resistance mechanisms in castration-resistant prostate cancer. Clin Cancer Res 2016;22:4466-4477.

65. Solit DB, Scher HI, Rosen N: Hsp90 as a therapeutic target in prostate cancer. Semin Oncol 2003;30:709-716.

66. Lievens $S$, Lemmens I, Tavernier J: Mammalian two-hybrids come of age. Trends Biochem Sci 2009;34:579-588.

67. Mendonca DB, Mendonca G, Cooper LF: Mammalian two-hybrid assays for studies of interaction of p300 with transcription factors. Methods Mol Biol 2013;977:323-338.

68. Stynen B, Tournu H, Tavernier J, Van Dijck P: Diversity in genetic in vivo methods for protein-protein interaction studies: from the yeast two-hybrid system to the mammalian split-luciferase system. Microbiol Mol Biol Rev 2012; 76:331-382.

69. Ravasi $T$, Suzuki $H$, Cannistraci CV, et al.: An atlas of combinatorial transcriptional regulation in mouse and man. Cell 2010;140:744-752.

70. Xu J, Wu RC, O'Malley BW: Normal and cancer-related functions of the p160 steroid receptor co-activator (SRC) family. Nat Rev Cancer 2009;9: 615-630.

71. Lonard DM, O'Malley BW: Molecular pathways: targeting steroid receptor coactivators in cancer. Clin Cancer Res 2016;22:5403-5407.

72. Agoulnik IU, Vaid A, Bingman WE, 3rd, et al.: Role of SRC-1 in the promotion of prostate cancer cell growth and tumor progression. Cancer Res 2005;65: 7959-7967.

73. Fujimoto N, Mizokami A, Harada S, Matsumoto T: Different expression of androgen receptor coactivators in human prostate. Urology 2001;58:289294. 
FANCHER ET AL.

74. Conn PJ, Christopoulos A, Lindsley CW: Allosteric modulators of GPCRs: a novel approach for the treatment of CNS disorders. Nat Rev Drug Discov 2009; 8:41-54.

75. Baell JB, Holloway GA: New substructure filters for removal of pan assay interference compounds (PAINS) from screening libraries and for their exclusion in bioassays. J Med Chem 2010;53:2719-2740.

76. Irvine RA, Ma H, Yu MC, Ross RK, Stallcup MR, Coetzee GA: Inhibition of p160mediated coactivation with increasing androgen receptor polyglutamine length. Hum Mol Genet 2000;9:267-274.

77. Ma $H_{1}$ Hong $H_{1}$ Huang SM, et al.: Multiple signal input and output domains of the 160-kilodalton nuclear receptor coactivator proteins. Mol Cell Biol 1999;19: 6164-6173.

Address correspondence to: Paul A. Johnston, PhD Department of Pharmaceutical Sciences School of Pharmacy University of Pittsburgh 700 Technology Drive Pittsburgh Technology Center (PTC), Room 4101 Pittsburgh, PA 15219

E-mail:paj18@pitt.edu

Abbreviations Used

$\%$ CVs $=$ percent coefficient of variance

17-AAG = 17-allylaminogeldanamycin

ADT $=$ androgen deprivation therapy

AF-1 = activation function 1

AF-2 = activation function 2

$\mathrm{AR}=$ androgen receptor

AR-NTD $=\mathrm{N}$-terminal domain of $\mathrm{AR}$

$\mathrm{ARE}=$ androgen response element

AR-V7 = androgen receptor splice variant 7

CPMs = counts per minute

$\mathrm{CRPC}=$ castration-resistant prostate cancer

$\mathrm{DBD}=$ DNA-binding domain

$\mathrm{DHT}=$ dihydrotestosterone
DMEM/F12 = Dulbecco's modified Eagle medium: nutrient mixture F-12

DMSO $=$ dimethyl sulfoxide

$\mathrm{FBS}=$ fetal bovine serum

$\mathrm{FL}=$ full length

Gal4 = galactose 4 DNA binding domain

GFP $=$ green fluorescent protein

$\mathrm{H}^{3}$-DHT $=$ tritiated dihydrotestosterone

$\mathrm{HCS}=$ high-content screening

HEK 293 = human embryonic kidney 293 cells

$\mathrm{Hsp}=$ heat shock protein

$I_{50}=50 \%$ inhibition concentration

IXM = ImageXpress Micro

$\mathrm{LBD}=$ ligand-binding domain

Luc $=$ luciferase

$\mathrm{LXXLL}=$ coactivator leucine-rich binding motifs

$\mathrm{M} 2 \mathrm{H}=$ mammalian 2-hybrid

$\mathrm{NCl}=$ National Cancer Institute

$\mathrm{NID}=$ nuclear interaction domain

$\mathrm{NR}=$ nuclear receptor

PAINS $=$ pan-assay interference compounds

PBS $=$ phosphate-buffered saline

$\mathrm{PCa}=$ prostate cancer

$\mathrm{PCR}=$ polymerase chain reaction

pGal4 = DNA-binding domain of the Gal4 protein derived from yeast

$\mathrm{PI}=$ principal investigator

$\mathrm{PPI}=$ protein-protein interaction

$\mathrm{PPIB}=$ protein-protein interaction biosensor

PSA $=$ prostate-specific antigen

RFP $=$ red fluorescent protein

$\mathrm{RLUS}=$ relative light units

$\mathrm{RT}=$ room temperature

$\mathrm{S}: \mathrm{B}=$ signal-to-background

$\mathrm{SA}-\mathrm{DB}=$ streptavidin donor beads

SFM = serum-free media

$\mathrm{SRC}=$ steroid receptor coactivator

TAU $5=$ transactivation unit 5

$\mathrm{TE}=$ translocation enhanced

TIF2 $=$ transcriptional intermediary factor 2

$\mathrm{U}-2$ OS $=\mathrm{U}-2$ osteosarcoma cell line

$\mathrm{UBE} 2 \mathrm{C}=$ ubiquitin-conjugating enzyme $\mathrm{E} 2 \mathrm{C}$ 